\title{
Mid-fidelity simulations and comparisons of five techniques for axial induction control of a wind turbine
}

\author{
Dan Houck $^{1}$, David Maniaci ${ }^{1}$, and Chris L. Kelley ${ }^{1}$ \\ ${ }^{1}$ Sandia National Labs, 1515 Eubank Blvd., SE, Albuquerque, NM 87123 \\ Correspondence: Dan Houck (drhouck@sandia.gov)
}

\begin{abstract}
As wind turbines are more frequently placed in arrays, the need to understand and mitigate problems arising from their wakes has increased. When downstream turbines are in the wakes of upstream ones, the downstream turbines produce less power, require more maintenance, and have shorter lifetimes. One wake mitigation technique is known as axial induction control (AIC) and it involves derating (operating suboptimally) upstream turbines such that more energy remains in their wakes

5 for downstream turbines to harvest. While there has been considerable research on this technique, much of it has suffered from a misunderstanding of the most important parameters in optimizing AIC. As such, the research has been largely inconclusive. Herein, we seek to rectify several perceived shortcomings of previous work by using mid-fidelity simulations to compare five different techniques for AIC at three different derate percentages against a baseline case and examining the recovery of the wake. We find that only the case with the lowest derate, $10 \%$, and using maximum thrust exceeds the baseline when estimating the combined power of the simulated turbine and a virtual turbine five diameters downstream and that it produced $10 \%$ more power. Furthermore, these results help to validate previous work that concluded that the excess energy that is in the wake of a derated turbine will be at the edges of the wake unless the wake can sufficiently recover before the next downstream turbine. Finally, all together this suggests that the precise combination of derate percentage and the method used to derate turbines (i.e., the precise combination of pitch and torque controls), as well as the spacing and arrangement of turbines, must all be considered when optimizing AIC, and that substantial power gains may be possible.
\end{abstract}

\section{Introduction}

As wind energy progresses, attention has shifted from optimizing individual turbines to optimizing wind farms to mitigate the negative effects of upstream turbines' wakes. In addition to turbine siting, there has been very promising research into coordinating turbine controls within a wind farm to maximize its total power. These methods have been recently and well-reviewed in Houck (2021) and Kheirabadi and Nagamune (2019). Axial induction control (AIC) is one method that has been heavily researched, and it involves derating upstream turbines for the benefit of downstream turbines. By reducing the power production of upstream turbines, the effects of their wakes are mitigated and downstream turbines produce more power as a result. The net result of this is an increase in total power of the multi-turbine system.

Previous research into AIC has produced mixed results and has been largely inconclusive as to its effectiveness. Some of the ambiguity of the results of previous studies can be attributed to one or more of three particular criticisms: the use of low-fidelity 
wake models, testing with columns and small arrays of turbines that are aligned with the inflow, and the oversimplification of turbine controls. First, low-fidelity models approximate, or simply do not include, wake dynamics that are known to be important to the wake decay process. For example, the Katic-Jensen model approximates the velocity deficit in the wake as a "top-hat" profile rather than radially varying (Katic, I., Hojstrup, J., Jensen, 1986). Furthermore, this and other wake models do not produce any differences in the wake, such as rotor-added turbulence, based on different thrusts and may not capture the effects of different levels of turbulence in the inflow.

Annoni et al. (2015) demonstrated these differences by comparing the high-fidelity large eddy simulation (LES) code SOWFA (Churchfield and Lee, 2012) and the engineering wake model code FLORIS (NREL, 2020). By looking at the wake of a derated turbine with each method, they demonstrated that the wake model did not capture the true spatial variation of energy within the wake while the LES code showed that the energy not harvested by a derated turbine is located primarily at the edges of its wake and not within it. A turbine directly downstream of a derated turbine is not optimally placed to benefit from the excess energy in the upstream wake.

This is exemplary of the second shortcoming of previous studies: they frequently use a column or small array of turbines aligned with the inflow. This arrangement creates a worst-case scenario for a baseline case such that one might expect that differences from using AIC would be more easily identified. Instead, it also creates a worst-case scenario for AIC since downstream turbines are likely in the worst possible places to benefit from the derating of upstream turbines. Downstream turbines that are aligned with the inflow are subjected to full wakes under conventional operation, and, when using AIC, they may receive little relief. In a large enough array, this may be mitigated depending on the array size and spacing as wakes continue to expand and mix downstream allowing turbines multiple rows downstream of derated turbines to benefit from the excess energy in their wakes.

Finally, many previous studies do not differentiate among the multiple combinations of controls that may achieve the same power production. Figure 1 shows an example of multiple derate strategies that achieve the same power. The " + " marks the turbine's typical operating point for a given wind speed and the ring around it is a contour of a constant, lower power. There is an infinity of combinations of pitch and tip speed ratio (TSR) that can produce this derate. Low-fidelity models, and even some higher fidelity methods, only provide details of the target power for the derate or an alternative such as the target thrust or induction. How the derate is achieved affects the qualities of the wake that is produced, so this information is vital to understanding the success or failure of an AIC method.

Annoni et al. (2015), for example, qualitatively compared the effects of increasing or decreasing the thrust to derate a turbine. While a lower thrust reduces the velocity deficit and leaves higher velocity in its wake for downstream turbines, the reduction in thrust also reduces the induced turbulence, which in turn decelerates the wake decay. Higher thrust produces lower velocities in the wake, but increases the induced turbulence and accelerates wake decay. The difference in the net effect of these may be null. Several studies, however, have arrived at the conclusion that minimizing the thrust when derating is most effective in terms of total array power (Mirzaei et al., 2015; Pedersen and Larsen, 2020; Schaak, 2006; Vitulli et al., 2019).

This study aims to address these challenges in researching AIC by using a mid-fidelity free vortex wake method (FVWM) to 
https://doi.org/10.5194/wes-2021-122

Preprint. Discussion started: 19 November 2021

(c) Author(s) 2021. CC BY 4.0 License.

(c) (i)
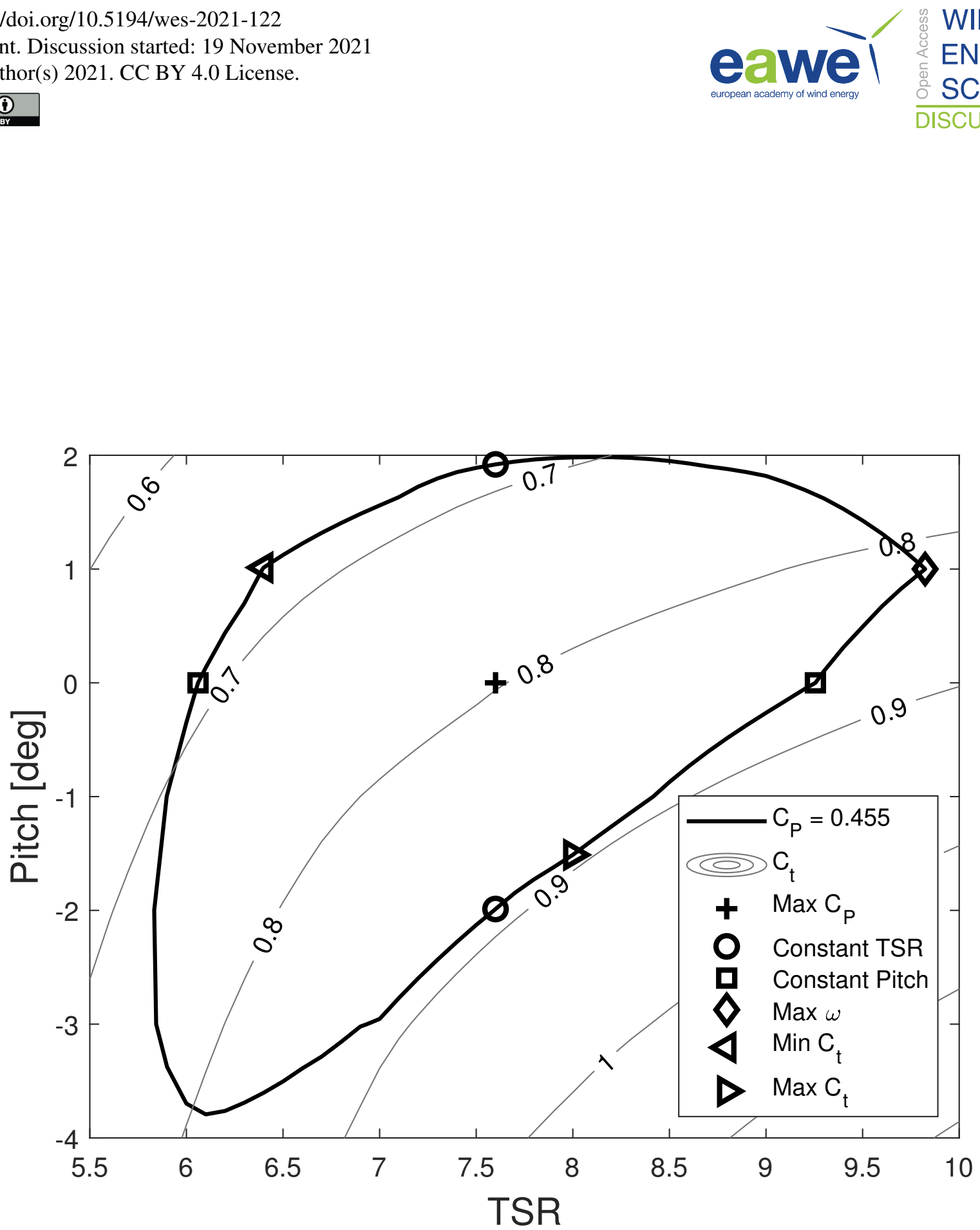

Figure 1. When considering a fixed derate, there are infinite ways to achieve it in combinations of pitch and TSR. This contour curve of constant $C_{P}$ as a function of pitch and $T S R$ shows five possible ways of achieving it when derating from the maximum $C_{P}$. Data from (Kelley, 2015). 
methods at computational speeds that are much faster than LES or direct numerical simulations (DNS) and can include the effects of a turbine's pitch and TSR, and so its induction and thrust as well. To further investigate the multiple pathways to a given power target when derating, we evaluated the differences in the wake when using five different strategies to achieve derates of $10 \%, 20 \%$, and $40 \%$ of the baseline power production: constant pitch, constant TSR, maximum rotation rate, minimum thrust, and maximum thrust. When considering the simulated turbine and a virtual downstream turbine, our results indicate that maximizing the thrust of the derated turbine yields the highest combined power of the two, though the placement of the downstream turbine is an important consideration in choosing a derate method.

The remainder of the article is organized as follows: In Section 2, details of the FVWM code, the turbine used, the simulation set up, and careful consideration of the code's limitations are provided. In Section 3, we provide results of the 16 simulations that were performed, and in Section 4 we evaluate the relevant differences in the wakes created by different derates and derate methods. Finally, conclusions and recommendations for future work are given in Section 5.

\section{Methods}

\subsection{Simulation Setup}

This study used the Code for Axial and Cross-flow TUrbine Simulation (CACTUS), a mid-fidelity FVWM developed by Sandia National Labs (SNL), to simulate a turbine in a three-dimensional domain. The turbine's blades are represented by discretized blade elements with lookup tables of two-dimensional airfoil polar data. The Kutta-Joukowski theorem relates the lift data to the bound vorticity at each blade element and the Helmholz theorem recovers the spanwise and trailing vorticities. A potential flow model uses straight line vortex elements to represent the wake and the induced velocities are calculated via the Biot-Savart law. CACTUS does not attempt to approximate viscous processes as is common in other FVWMs (Ananthan et al., 2002; Leishman et al., 2002; Marten et al., 2020, 2016), so the vortex filaments are advected with the freestream flow without dissipation or diffusion, though regularization is implemented with a cut-off radius for the vortex cores. More complete descriptions of CACTUS are given in (Kelley et al., 2015; Murray and Barone, 2011; Murray et al.; Wosnik et al., 2016).

Extensive test cases were run in CACTUS to verify the best combination of many settings to ensure as accurate results as the tool allows. Performance coefficients of power, torque, and thrust are within $1 \%$ of their converged values within only approximately 20 revolutions for the majority of simulations, but wake statistics require more revolutions. All simulations were run for 45 revolutions and the velocity data from the last 15 revolutions is saved for analysis based on convergence tests of many of the wake statistics used in section 3 . The number of time steps per revolution was also given careful consideration. Bhagwat and Leishman (2000) showed in their FVWM code that increments of five degrees or less were required to achieve second order accuracy. Test cases with CACTUS indicated that 50 time steps per revolution, or $7.2^{\circ}$, achieved convergence of wake statistics, but 72 time steps per revolution, or $5^{\circ}$, was used to ensure greater accuracy and to help with the stability of the solution by reducing the change in blade loads between timesteps.

CACTUS allows for user selection of either no vortex core model, a constant one, or a linear one. It was observed that having no core model accelerated wake recovery while the constant and linear core models produced wakes that looked fairly similar 


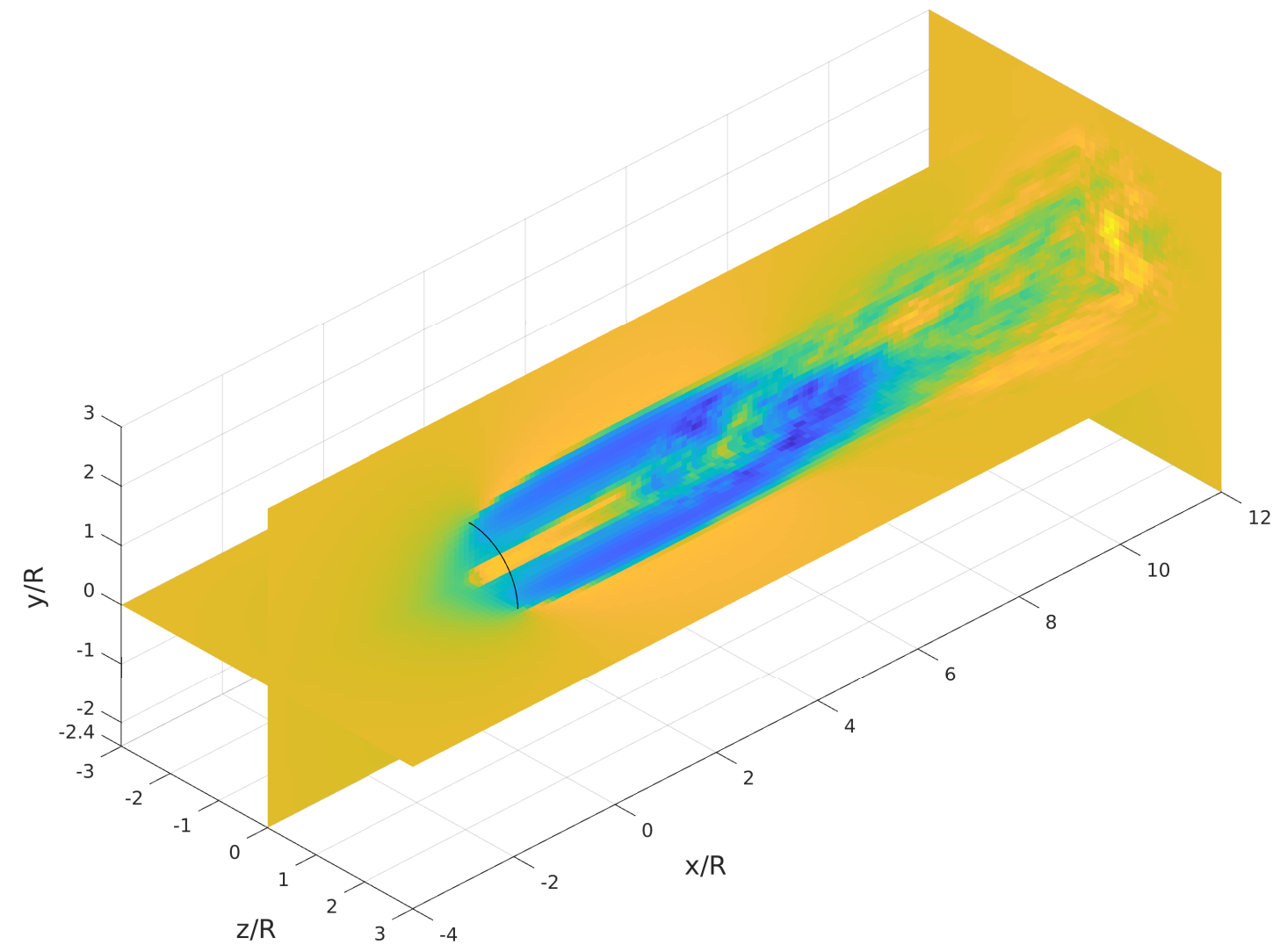

Figure 2. The coordinate system and saved domain are shown with slices through the Baseline simulation horizontally at hub height, vertically through the center, and the outlet plane. A black line shows a quarter of the rotor-swept perimeter at $x / R=0$.

to each other. The linear core model was chosen because it is a closer approximation to the viscous core of a real vortex and because it showed a slightly slower, and so more conservative, wake recovery rate. In all of these models, regularization is performed using a cut-off radius to avoid the singularity of the core at zero radius. This was left at the default of $10^{-7}$. Defaults were also used for the initial vortex core sizes, which are set as the maximum chord for the bound vorticity, the maximum blade element span for the trailing vorticity, while the core size for the spanwise vorticity is calculated from a reference distance between spanwise wake lines given the temporal discretization level (Murray et al.). These initial core sizes are in line with recommendations from Xu et al. (2019) who suggest keeping them above half the blade chord to ensure the power coefficient is accurately predicted. Time step filtering was used to smooth bound vorticity over three timesteps in all simulations as it was found necessary for stability during simulations with high $T S R$. 
The simulated turbine is the National Rotor Testbed (NRT) rotor designed by SNL to fly on a modified V27 turbine while replicating the scaled wake of a 1.5 MW turbine (Kelley et al., 2016). This turbine is part of the Scaled Wind Farm Test facility (SWiFT) operated by SNL at the Reese Technology Center in Lubbock, TX (Berg et al., 2013). The rotor has a diameter of $27 \mathrm{~m}$ with a $4^{\circ}$ shaft up-tilt and a hub height of $32 \mathrm{~m}$. The CACTUS software includes several Matlab scripts for creating the necessary geometry files by inputting blade data and setting the desired pitch. Each blade was discretized into 42 uniformly distributed elements using nine different sets of polars that are provided to CACTUS as lookup tables over a range of Reynolds numbers. Polars for the lookup tables were generated in QBlade (Marten et al., 2013), which integrates XFOIL for the calculation of two-dimensional airfoil data and allows for extrapolation of polar data to 360 degrees with the Montgomerie method (Montgomerie, 2004). The Leishman-Beddoes dynamic stall model is implemented in CACTUS to account for any transient stall effects (Leishman and Beddoes, 1989). It should be noted that the turbine's nacelle, including the hub, are not simulated and result in an unrealistic jet in the middle of the near-wake. A tower model adds an empirically defined wake deficit to the freestream flow to model the presence of the tower.

For purposes of the code's calculations, the turbine is in an infinite domain. The velocity field was saved over a domain stretching two diameters (D) upstream of the turbine, 6D downstream, and 1.5D in either spanwise direction and upward as seen in Fig. 2. After 24D downstream of the turbine, the vortex elements are ignored in the induced velocity calculations to facilitate faster computation. Based on a grid sensitivity study, the velocity data are computed and saved on a grid with spacing of $0.05 \mathrm{D}$ in all directions.

The inflow is the same in all cases. CACTUS does not provide methods for a turbulent inflow. Simulating a sheared inflow using a passive inflow model results in non-physical lifting of the turbine wake (Branlard et al., 2015). The inflow is uniform, laminar, and steady at $6.8 \mathrm{~m} / \mathrm{s}$, the density of air is $1.08 \mathrm{~kg} / \mathrm{m}^{3}$, and its dynamic viscosity is $1.8 \times 10^{-5}$ (used for calculation of the Reynolds number and selection of the appropriate airfoil polar), which are the annual mean wind speed, density, and viscosity of air at the SWiFT site (Kelley and Ennis, 2016).

\subsection{Simulation Cases}

Five different derate strategies were simulated with three different derates for a total of 16 simulations including a Baseline case during which the turbine is operating at its rated power for the freestream wind speed. During this simulation, the turbine operates with a pitch of zero degrees and a $T S R$ of nine. The remaining cases can be seen in Table 1 and are plotted in Fig. 3 on top of contours of $C_{P}$ and $C_{t}$. It should be noted that, in theory both the constant pitch and constant TSR cases present two options: an increase or decrease. For the constant pitch cases, the the TSR had to be decreased as an increase would have exceeded the turbine's maximum rotation rate. For the constant $T S R$ cases, a higher pitch was chosen as it maintains a positive angle of attack on the airfoil for which performance is more accurately predicted when calculating polars, which should aid in the accuracy of the simulations.

The three derates were chosen to extend over a wide range of possibilities. In a deep array, it is possible (though it remains to be determined what is optimal) that the most upstream turbines would require significant derates to maximize the array power. 


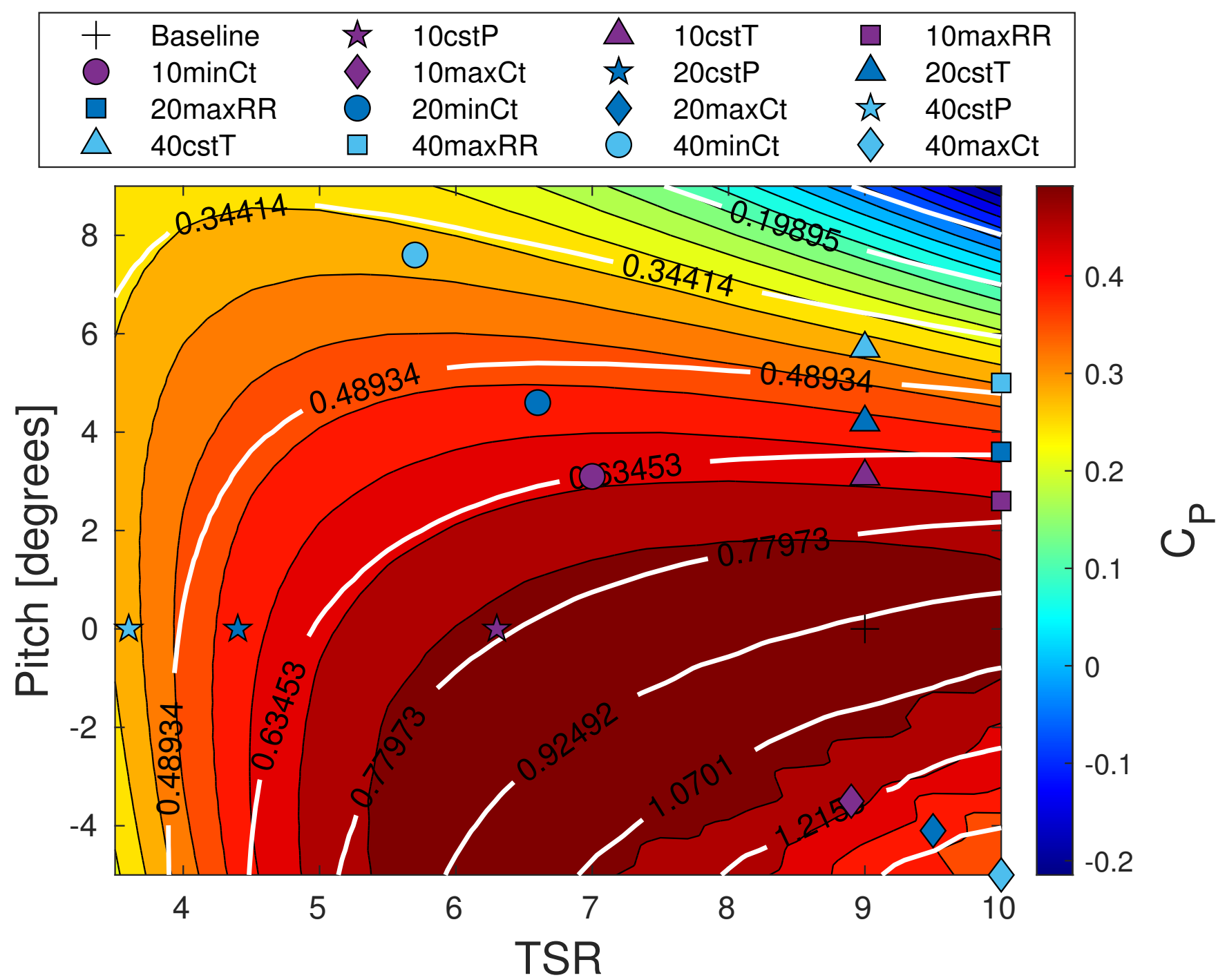

Figure 3. The target $T S R$ and pitch for each case listed in Table 1 is plotted along with the Baseline on top of contours of $C_{P}$ with contours of $C_{t}$ (white lines) and their values overlaid. 
Table 1. Table of the pitch and $T S R$ used for each simulation case. The derate indicates the percent decrease in power from the Baseline.

\begin{tabular}{l|rr|rr|rr}
\hline Derate $\rightarrow$ & \multicolumn{2}{|c|}{$\mathbf{1 0 \%}$} & \multicolumn{2}{c|}{$\mathbf{2 0 \%}$} & \multicolumn{2}{c}{$\mathbf{4 0 \%}$} \\
\hline Case $\downarrow$ & Pitch & TSR & Pitch & TSR & Pitch & TSR \\
\hline Constant Pitch (cstP) & 0.0 & 5.27 & 0.0 & 4.40 & 0.0 & 3.60 \\
Constant TSR (cstT) & 3.16 & 9.00 & 4.15 & 9.0 & 5.66 & 9.00 \\
Max Rot. Rate (maxRR) & 2.59 & $10.12^{1}$ & 3.61 & 10.12 & 4.99 & 10.12 \\
Min $C_{T}$ (minCt) & 3.14 & 6.92 & 4.64 & 6.58 & 7.63 & 5.74 \\
Max $C_{T}$ (maxCt) & -3.50 & 8.90 & -3.90 & 9.30 & -5.00 & 10.12
\end{tabular}

\footnotetext{
${ }^{1}$ This is the maximum TSR at the simulated freestream wind speed.
}

The five strategies considered were chosen for the following reasons: Keeping pitch or TSR constant while derating yields an easier control option and could reduce wear on components if frequent adjustment is required. The idea of maximizing the rotation rate for a given derate comes from literature studying the breakdown of tip vortices (Houck and Cowen, 2019; Iungo et al., 2013; Ivanell et al., 2010; Lignarolo et al., 2015). These studies have found that when turbines operate at higher rotation rates, it reduces the pitch (streamwise distance) between the tip vortex helices, and this causes them to interact and break down sooner. This breakdown accelerates wake mixing and decay. Finally, the reasoning behind minimizing or maximizing the thrust was reviewed above.

\subsection{Simulation Outputs}

As mentioned, the induced velocity is calculated at every timestep and output on a user-defined grid. This three-component velocity field is then used for further wake analyses as well as the turbine's coefficients of power, thrust, and torque. Care must be taken when examining turbine wakes simulated by FVWMs as CACTUS, in particular, has primarily been validated against experimental data for power and blade loads as opposed to wake data. In the near-wake, the individual vortex filaments remain neatly organized in helices and have been shown to accurately represent near wake dynamics in CACTUS (Ennis et al., 2015) and other FVWM codes (Corniglion et al., 2020; Marten et al., 2020, 2016). In the far-wake, however, and in particular after tip vortex breakdown, the filaments become highly disorganized and entangled and create a sort of numerical turbulence. Physically, there is a feedback loop in which vortex-vortex interactions cancel the spanwise and vertical components of the induced velocity, which causes the vortices to align more in the streamwise direction with varying orientations such that the spanwise and vertical induced velocity components further cancel and result in apparent wake recovery. This leads to an increasingly streamwise-dominant flow, though it may be impossible to distinguish between physical and numerical instabilities and dissipation (Bhagwat and Leishman, 2001). The calculations performed by CACTUS are explicitly inviscid, i.e., there is no diffusion or dissipation, so solutions become less physically realistic in proportion to the wake age or its distance downstream, but this is difficult to quantify without comparison to validation data. Wind turbine wake decay, however, is a largely inviscid 
process (large scale turbulence is essentially inviscid), and dissipation is actually highest in the near-wake (Chamorro et al., 2014; Houck and Cowen, 2019). Since the effects of viscosity occur very close to surfaces and within the relatively small vortex cores, the bulk of the flow can be simulated by a potential flow model such as CACTUS.

To the author's knowledge, there is only one study that compares CACTUS to a higher fidelity method in the far-wake of a wind turbine. Ennis et al. (2015) compared CACTUS to the Virtual Wind Simulator (VWiS), an actuator line model LES code (Yang et al., 2015) simulating the same turbine and inflow conditions in both. Their results indicated that the velocity deficit profiles from CACTUS and VWiS match well up through 7D downstream and that the wakes demonstrate similar growth rates as defined by the radius that contains $95 \%$ of the mass deficit from the freestream. A standard error calculation on their timeaveraged streamwise velocities shows that they have an error of less than $10 \%$ between approximately 3D and 7D downstream and that it remains below 15\% between 0D and 8D. CACTUS, however, had greater Reynolds shear stress due to its lack of dissipation and, as a result, experienced a more rapid momentum recovery of its wake.

This last point suggests some caution in interpreting results herein as the turbine is operated in several distinct ways. Some of the test cases are likely to (indeed, intended to) create differences in wake recovery that are likely to mitigate or exacerbate the shortcomings of CACTUS depending on the levels of "turbulence" they generate. Despite the fact that CACTUS simulations are not as high fidelity as other methods, this is somewhat mitigated by the control and treatment approach. Any errors induced by the method relative to higher fidelity or experimental methods can likely be viewed as bias errors that are negligible when restricting results to differences among the cases. Additionally, the drastically lower computational cost of CACTUS relative to higher fidelity LES allows for the broader exploratory study presented here, which can be followed by higher fidelity methods to verify any promising results found herein.

\section{Results}

\subsection{Verification of simulations}

Before exploring the results related to the main objective, the data produced by CACTUS were used to verify it against theory in lieu of higher fidelity or experimental data. In particular, a control volume of energy was calculated as the primary concern in a simulation without viscosity is that energy in the domain will become imbalanced due to the lack of dissipation. The complete equation for the control volume of energy is given as

$185 \frac{\mathrm{d}}{\mathrm{dt}} \iiint_{V} E \mathrm{~d} V+\iint_{A}(\boldsymbol{U} E+\boldsymbol{T}) \cdot \boldsymbol{n} \mathrm{d} A=-\iiint_{V} 2 \nu S_{i j} S_{i j} \mathrm{~d} V-\dot{W}$,

where $E$ is energy; $V$ is the control volume; $\boldsymbol{U}$ is the velocity vector; $\boldsymbol{n}$ is the outward facing normal of each face of the control volume, $A ; \nu$ is viscosity; and $S_{i j}$ is the strain rate tensor. The transport, $\boldsymbol{T}$, is

$T_{i}=U_{i} p / \rho-2 \nu U_{j} S_{i j}$,

where $p$ is the fluid pressure, and $\rho$ is the fluid density. $\dot{W}$ is the work done by the turbine and is $\dot{W}=C_{P}(t) \frac{1}{2} U_{\infty}^{3} A_{T}$, 
where $C_{P}$ is the coefficient of power defined as

$C_{P}=\frac{P_{m e c h}}{0.5 \rho U_{\infty}^{3} A_{T}}$,

where $P_{\text {mech }}$ is the mechanical power produced by the turbine, $U_{\infty}$ is the freestream velocity, and $A_{T}$ is the turbine rotor-swept area. While it would not typically apply to flow through a wind turbine, the assumptions of the simulation, namely that it is inviscid, incompressible, and steady (in the mean), allow us to apply Bernoulli's Principle to calculate the pressure field at each time step, which is

$p=\frac{1}{2} \rho\left(U_{\infty}^{2}-U_{r e s}^{2}\right)$,

where $U_{\text {res }}$ is the resultant velocity at each point in the velocity field and is

$U_{\text {res }}=\sqrt{u^{2}+v^{2}+w^{2}}$,

where $u, v$, and $w$ are the instantaneous streamwise, transverse, and vertical velocities, respectively.

Ignoring viscous terms, the resulting energy balance is shown in Fig. 4. The first term of Eqn. 1 is the "Volume" and the second term results in a sum of energy flux for each surface of the control volume. The work done by the turbine is negligible compared to the other terms due its relatively small area compared to the control volume surfaces and its efficiency. It can be seen that, in an average sense, energy is fairly balanced in most simulations, with 10 maxCt being the largest exception due to a larger Volume term. The larger Volume terms indicate a lack of complete convergence as one would expect the temporally averaged time rate of change of the volume integral of energy to become constant and near-zero once a simulation had reached steady-state.

To investigate this further, the residual of the energy balance is plotted as a function of time for each case (except the Baseline) in Fig. 5. It is not shown, but by inspecting each term of the balance as a function of time, it is evident that the large fluctuations in the residual come almost entirely from the Volume term. There is a sense in Fig. 5 that the residual would converge to near-zero given enough time, but it is clear that the simulations are not all equally converged. Concern for this is mitigated by looking at Fig. 6, which shows the temporally averaged energy residual as a function of $x$. Here we see that the average residual never has a magnitude greater than $2 \%$ of the total energy. Within the confines of CACTUS, further convergence would most likely be achieved by simply simulating additional rotor revolutions.

Finally, the quality of the apparent turbulence generated by CACTUS can be evaluated by looking at the spectra of the centerline resultant velocity as a function of $x$. Under realistic flow conditions, we would expect to see a large inertial subrange and small dissipation subrange in the near-wake with the former decreasing and latter increasing as the wake progresses downstream (Chamorro and Porté-Agel, 2009). Figure 7 shows the spectra at $x / R=0,1,3,6$, and 12 and shows slopes of $-5 / 3$ and $-7 / 3$. The $-5 / 3$ slope indicates that CACTUS captures an inertial subrange in the apparent turbulence that is generated. The $-7 / 3$ slope, however, does not conform to any known turbulence theory for this type of flow and is a direct result of the non-physical lack of dissipation. The spectra at $x / R=0$ and 1 have little to no inertial subrange and are even flat in many cases indicating that nothing like turbulence has developed from vortex-vortex interactions yet. By $x / R=3$, most 
https://doi.org/10.5194/wes-2021-122

Preprint. Discussion started: 19 November 2021

(c) Author(s) 2021. CC BY 4.0 License.

european academy of wind energy

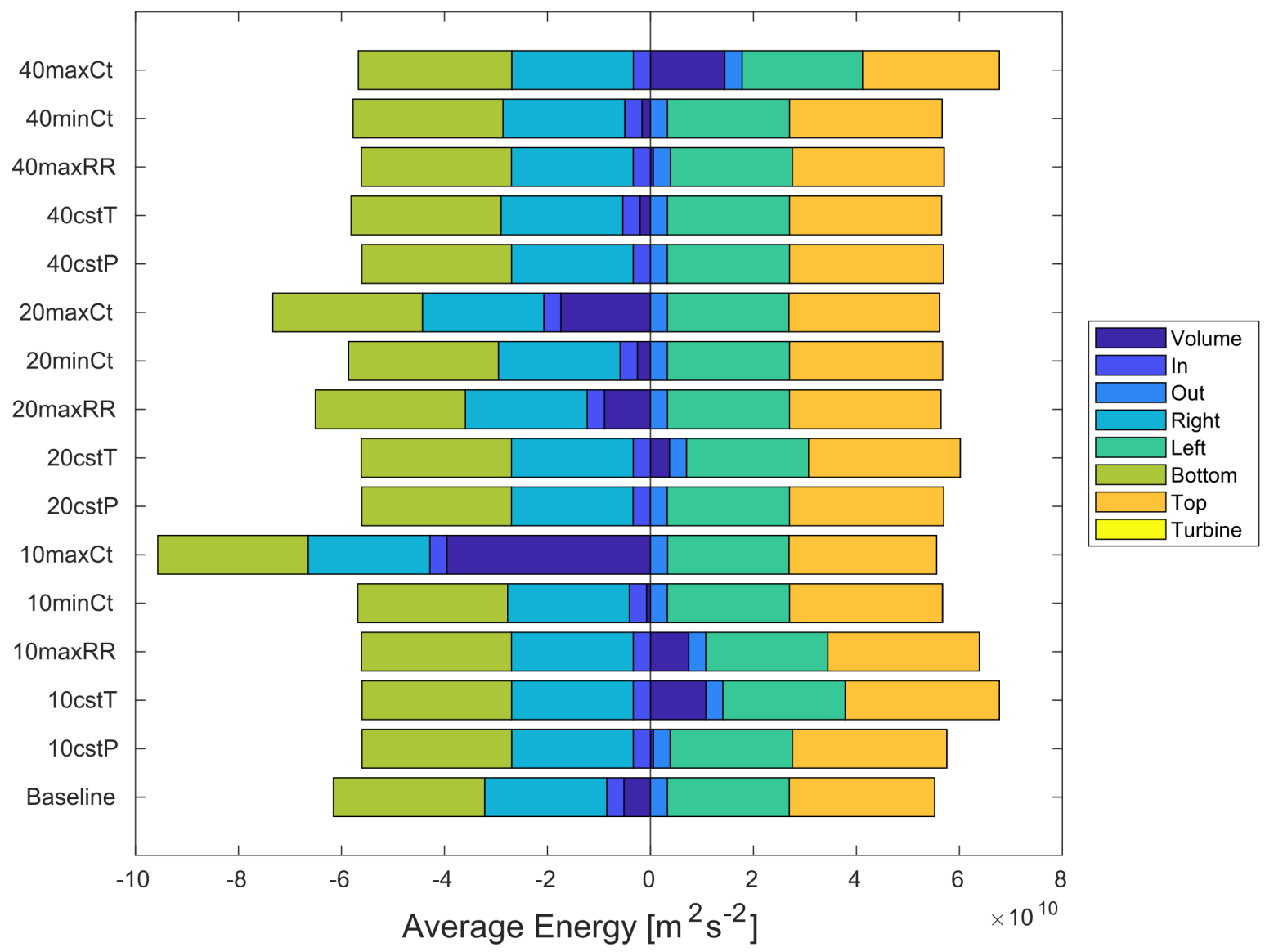

Figure 4. The near equality of the positive and negative energy terms as calculated from Eqn. 1 indicates that, on average, energy remains fairly balanced despite there being no dissipation in the simulations. Cases with higher thrusts are the exception to this and demonstrate a lack of convergence. 
https://doi.org/10.5194/wes-2021-122

Preprint. Discussion started: 19 November 2021

(c) Author(s) 2021. CC BY 4.0 License.

(c) (1)
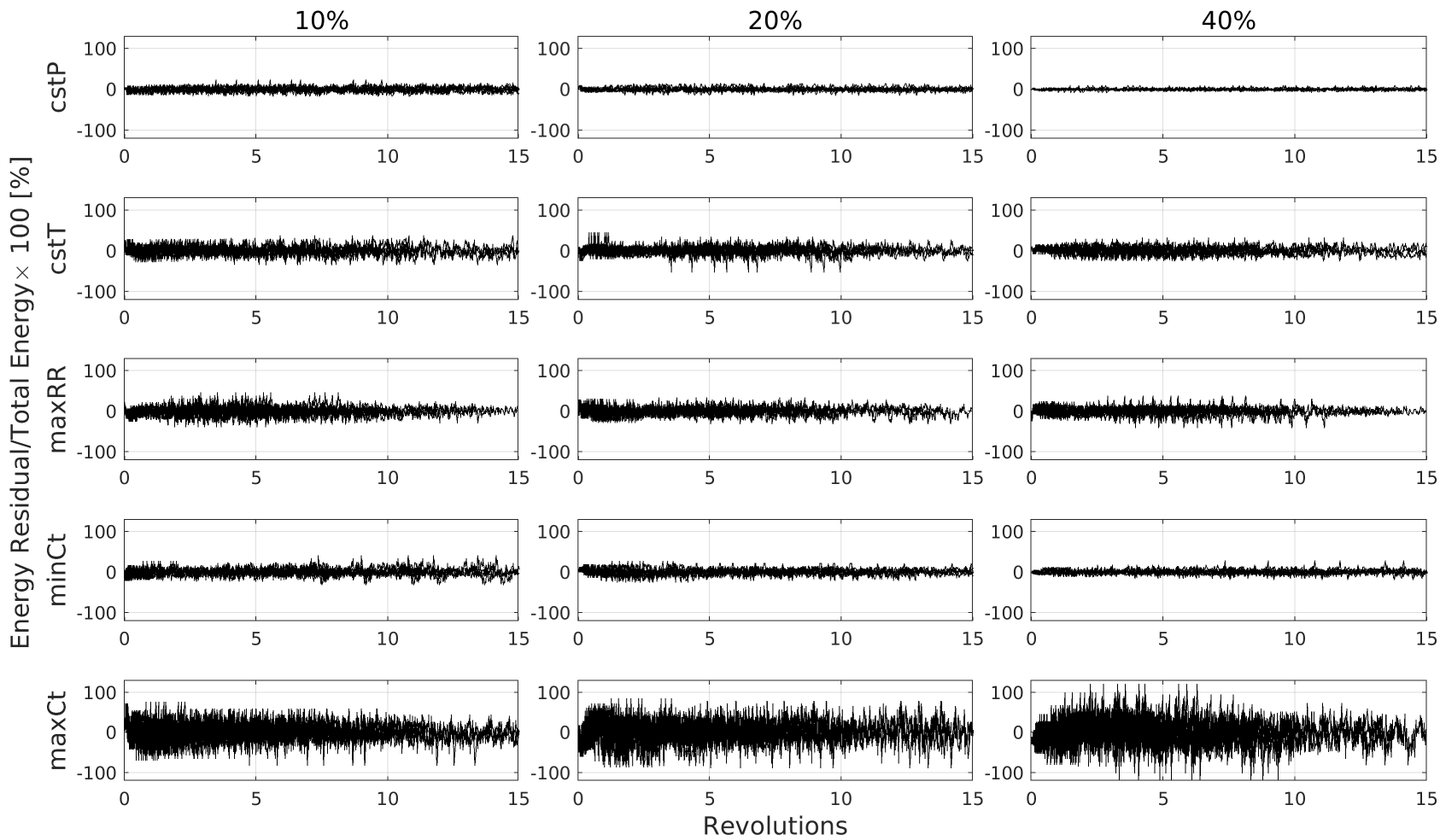

Figure 5. The residuals of the energy balances shown in Fig. 4 are plotted in time as percents of the total energy. They provide further evidence of the lack of convergence as the fluctuations come almost entirely from the Volume term.

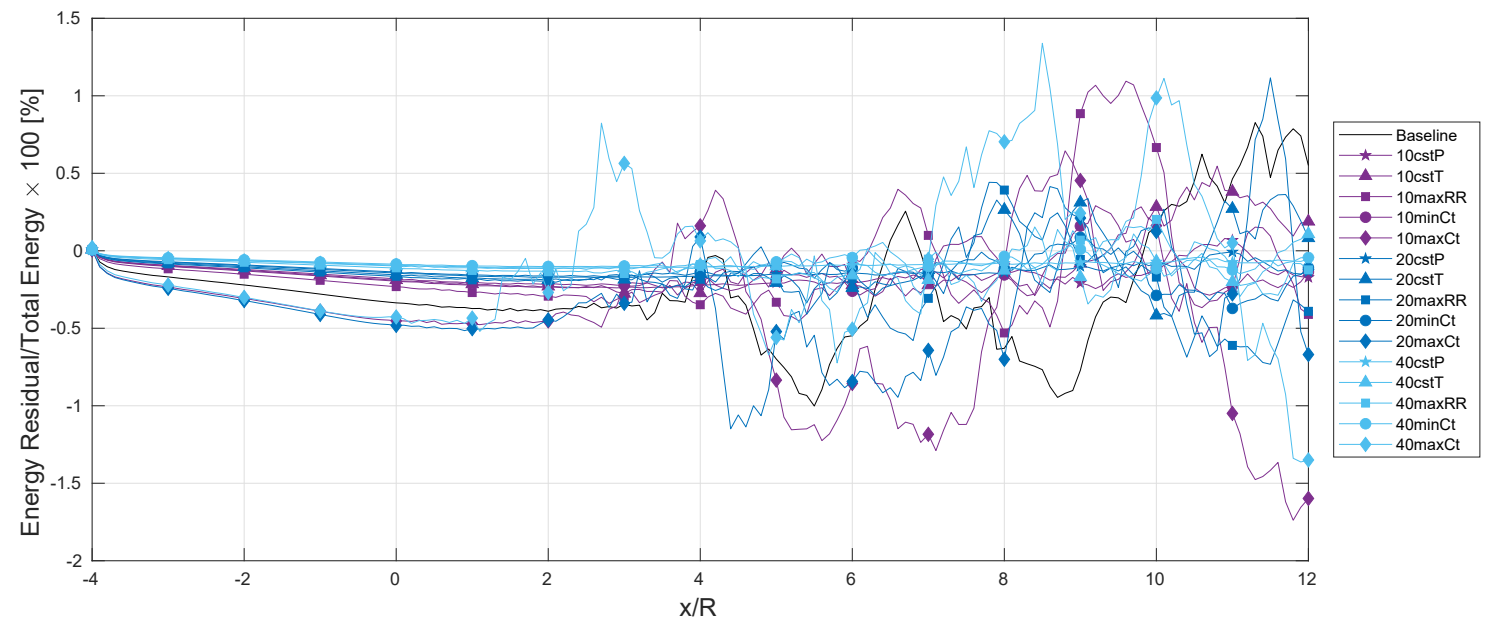

Figure 6. The temporally averaged energy residual as a function of $x$ shows that, on average, the residual remains small. 
https://doi.org/10.5194/wes-2021-122

Preprint. Discussion started: 19 November 2021

(c) Author(s) 2021. CC BY 4.0 License.
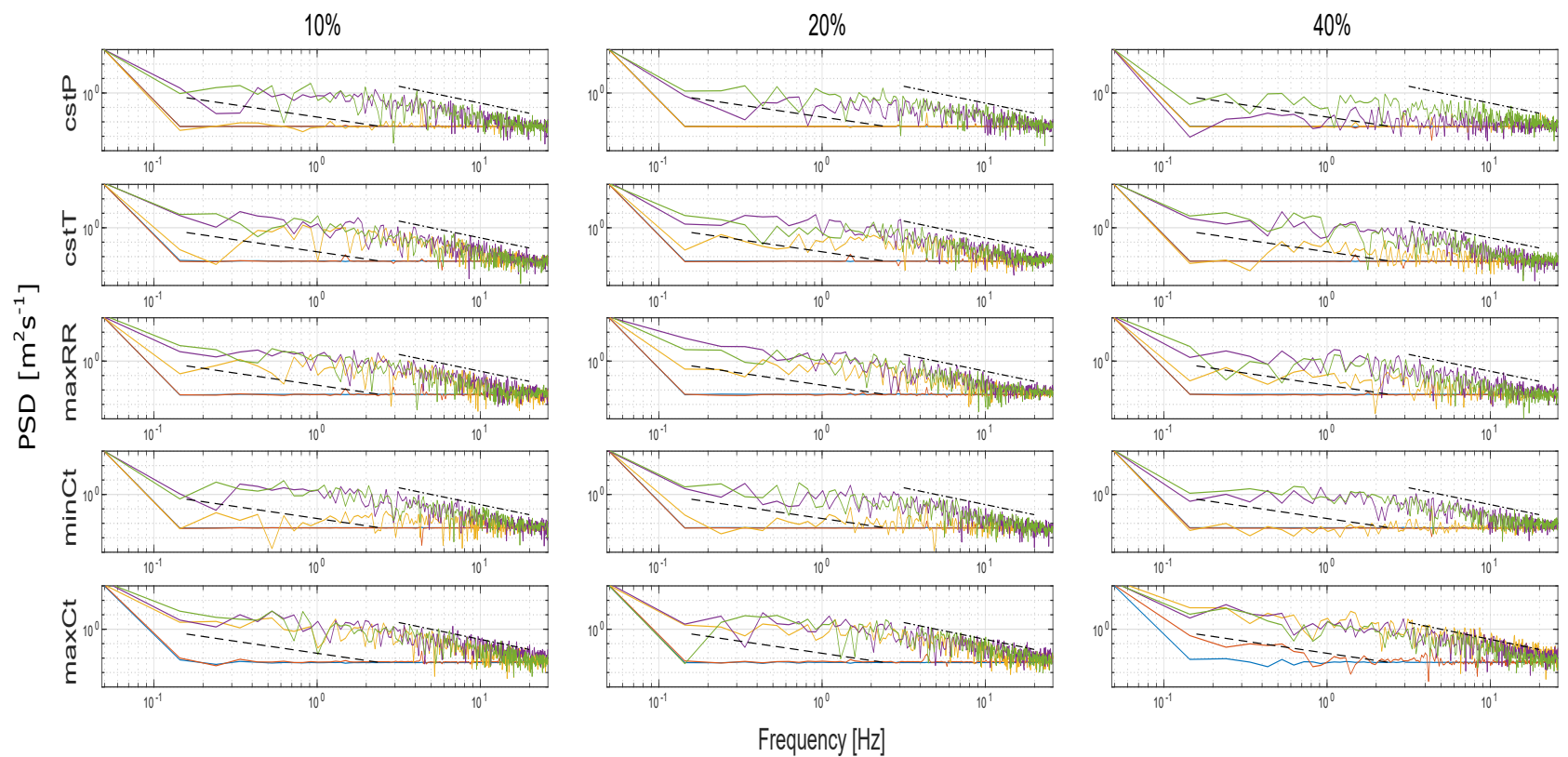

$x / R=0-x / R=1-x / R=3-x / R=6-x / R=12---5 / 3-\cdots--7 / 3$

Figure 7. Spectra of the centerline resultant velocity of each case plotted at different $x$ locations show the development of "turbulence" downstream.

cases begin to demonstrate an inertial subrange and by $x / R=12$, they all exhibit a $-7 / 3$ slope. This is likely the result of the flow developing smaller and smaller velocity fluctuations without any energy actually being dissipated while large scale turbulence is continuously generated, but this would need to be verified with further investigation. Furthermore, these results are within the wake, though any ambient turbulence is created by the induced velocities of vortex-vortex interactions of the wake. In a sense, the ambient turbulence develops along with the wake-generated turbulence.

\subsection{Qualitative results}

Moving on to results focused on the main objective, the wakes generated by each case are shown in Fig. 8. There are many ways to visualize wind turbine wakes (see (Quon et al., 2020)) and these results are only meant to be qualitative. The contours used to generate these wake visualizations were determined by the following steps: Contours of the temporally averaged streamwise velocity deficit calculated as $1-\bar{U} / U_{\infty}$ were created in each $y-z$ plane. These were then filtered to remove, first, open contours, then contours with areas smaller than the rotor-swept area, and then contours with a centroid coordinate that was more than $0.7 R$ from the center of the rotor-swept area. From the remaining contours in each plane, the contour level closest to zero was chosen corresponding to a more recovered (smaller deficit) wake. The red dot at the middle of each contour is the centroid of that contour and provides an indication of wake center. Looking down the columns of Fig. 8, we see that, for a 
https://doi.org/10.5194/wes-2021-122

Preprint. Discussion started: 19 November 2021

(c) Author(s) 2021. CC BY 4.0 License.

(c) (1)

eawe'

WIND

ENERGY

SCIENCE

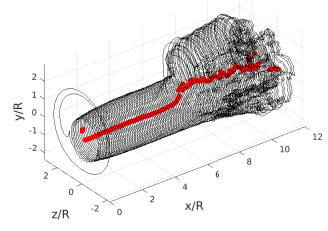

(a) Baseline

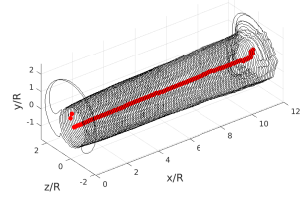

(b) $10 \mathrm{cstP}$

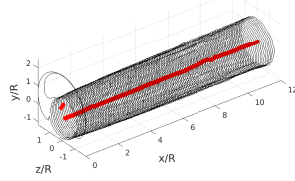

(g) $20 \mathrm{cstP}$

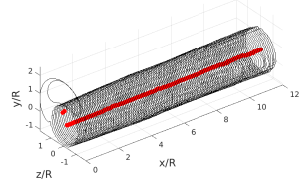

(1) $40 \mathrm{cstP}$

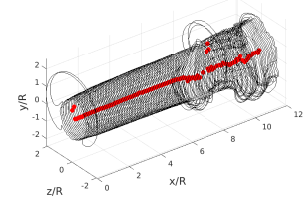

(c) $10 \mathrm{cst} \mathrm{T}$

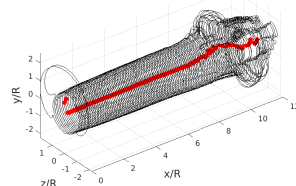

(h) $20 \mathrm{cst} \mathrm{T}$

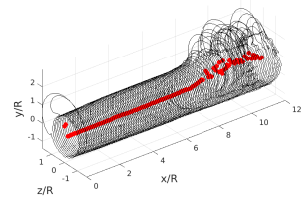

(m) 40cst T

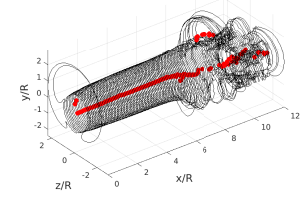

(d) 10maxRR

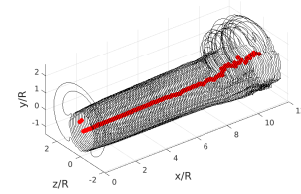

(e) $10 \mathrm{minCt}$

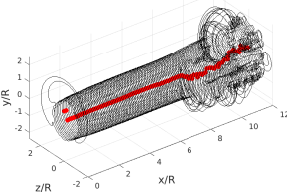

(i) $20 \max R \mathrm{R}$

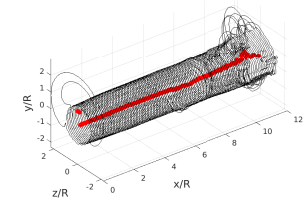

(n) $40 \mathrm{maxRR}$

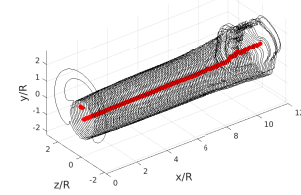

(j) $20 \mathrm{minCt}$

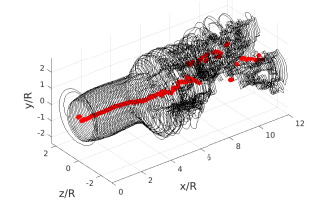

(f) $10 \mathrm{maxCt}$

Figure 8. Wake visualizations produced by filtering contours of the velocity deficit in each $y-z$ plane with red dots showing the centroid of each contour. These qualitatively indicate the differences in wake recovery in each case.

given derate technique, wake recovery happens more slowly for higher derates, though the difference can be subtle. This may be expected as smaller velocity deficits would create shear of a smaller magnitude and the shear-induced mixing is largely responsible for wake decay (Castillo and Newman, 2013; Chamorro et al., 2012). Already it appears that there might be an exception to this in the maxCt cases as the $40 \%$ derate appears to have the shortest near-wake of those cases. Looking across the rows, we can compare the different techniques for a given derate and here we see much larger differences. The maxCt cases stand out as those wakes appear to decay more quickly while the cstP cases barely decay at all.

Contours of the velocity deficit in the $x-z$ plane shown in Fig. 9 provide additional detail regarding the evolution of the wake in each case. In general, it appears that higher derates (lower power setpoints) produce less mixing and a slower wake recovery. Within the confines of these simulations, this is as expected because there are no turbulent motions until they are produced by vortex-vortex interactions. If there is a smaller velocity deficit, the flow remains more uniform and mixing is 

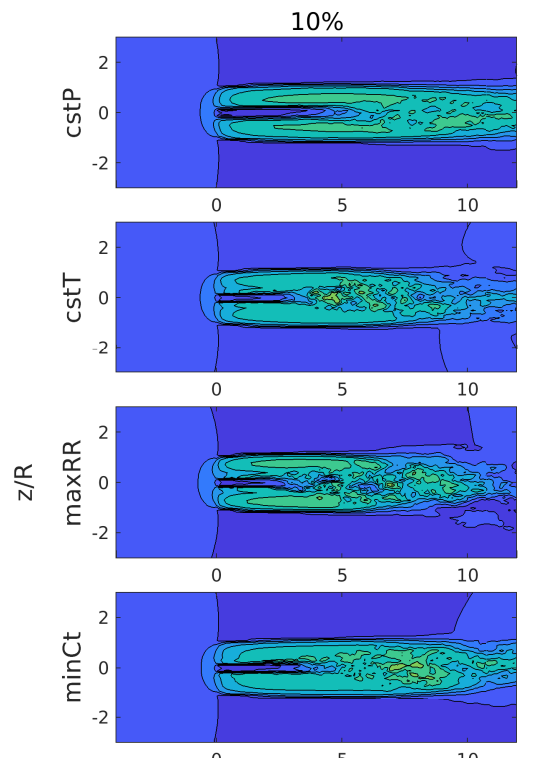

0

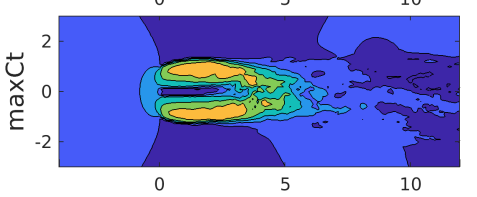

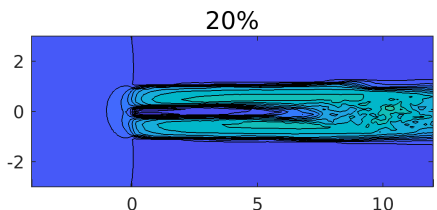
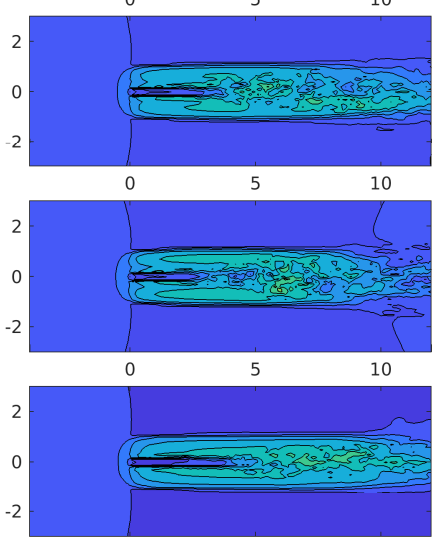

0

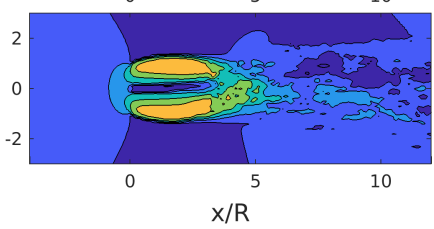

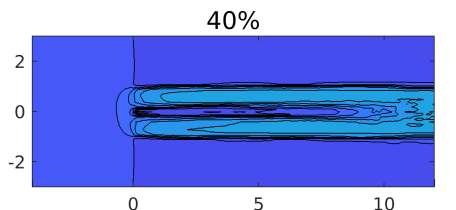
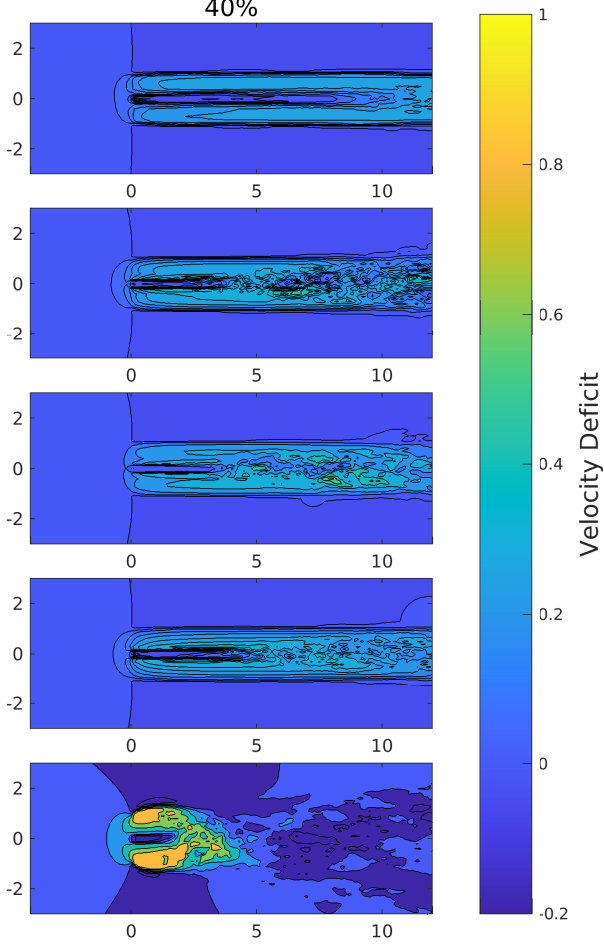

Figure 9. Contours of the velocity deficit in the hub height $x-z$ plane show some of the differences in flow produced by the different techniques and derates.

limited, which slows wake recovery. This is also seen to have the effect of stretching the region of high velocity (low velocity deficit) that appears where the rotor hub and nacelle would be. The maxCt cases have a behavior opposite of the others in that the highest derate (40\%) appears to recover faster than the lower derates. In these cases, there is a substantially larger deficit about the edges of the near-wake with a very sharp gradient to increased velocities in the ambient flow. The high thrust in these cases, which is higher for the higher derates, effectively creates a blockage behind the rotor leading to an acceleration of ambient flow around the near-wake. This area of high gradient produces rapidly growing shear (as shown below) and facilitates rapid mixing and wake recovery.

The time-averaged vertical vorticity is shown in Fig. 10. Tip vortices play an important role in wake decay as their highly stable nature tends to shield the wake from significant mixing until they destablize and decay at which point increased mixing between the wake and the ambient flow occurs (Brown et al., 2021; Houck and Cowen, 2019; Ivanell et al., 2010; Lignarolo et al., 2015). Simulating the evolution of the tip vortices is also one of the strengths of an FVWM. In addition to the vertical vorticity, a metric was created to track the strength of the vorticity along the lower side of the rotor in each subplot. First, at 
https://doi.org/10.5194/wes-2021-122

Preprint. Discussion started: 19 November 2021

(c) Author(s) 2021. CC BY 4.0 License.

(c) (1)
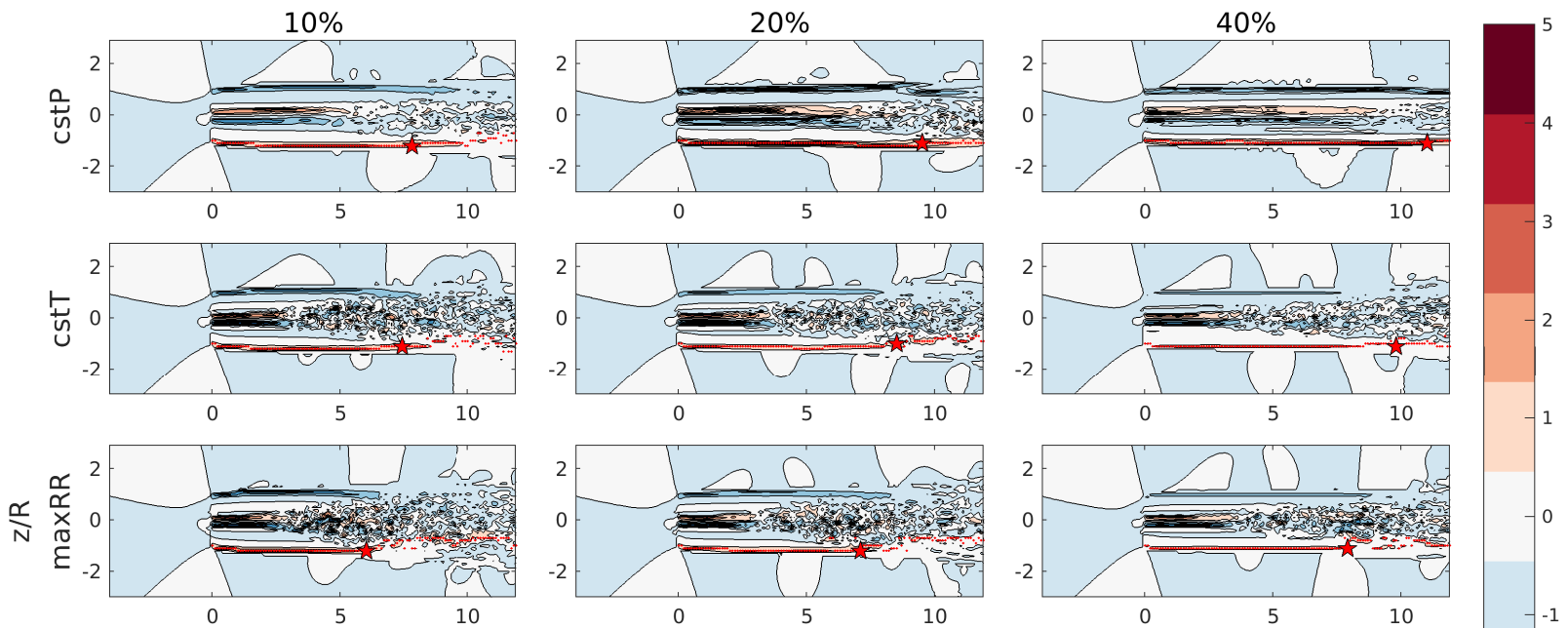

$1 \frac{.2}{.0}$
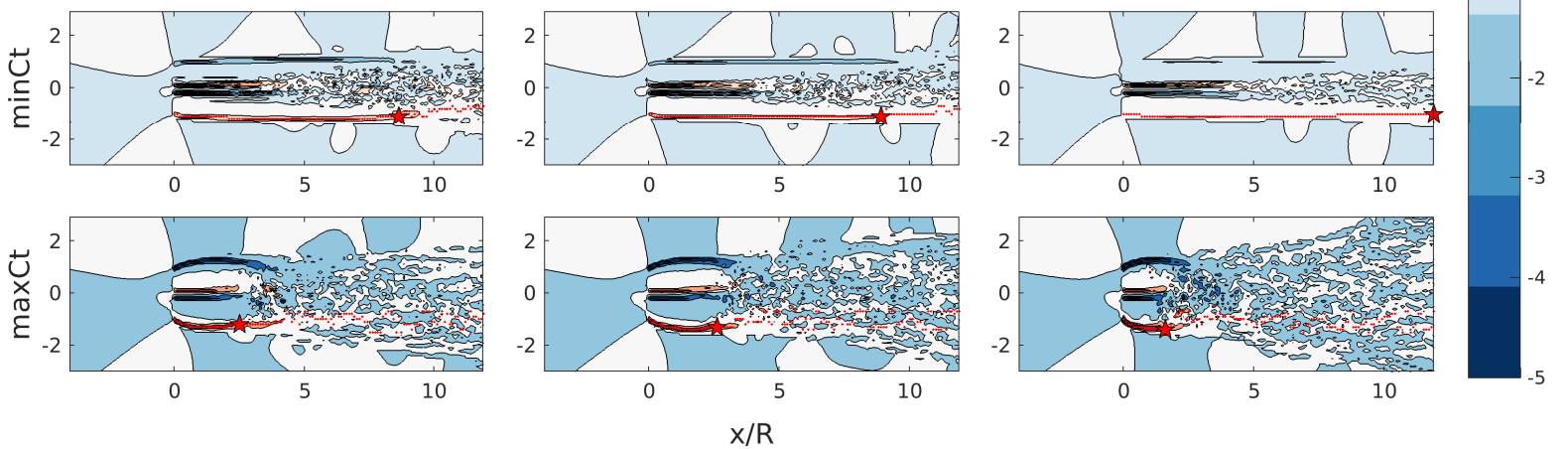

Figure 10. Contours of vertical vorticity for each case overlaid with the local maximum vorticity (red dots) at each $x$ location and the first time the local vorticity falls below $50 \%$ of the global maximum (red star). This shows the connection between vorticity breakdown and wake decay. 


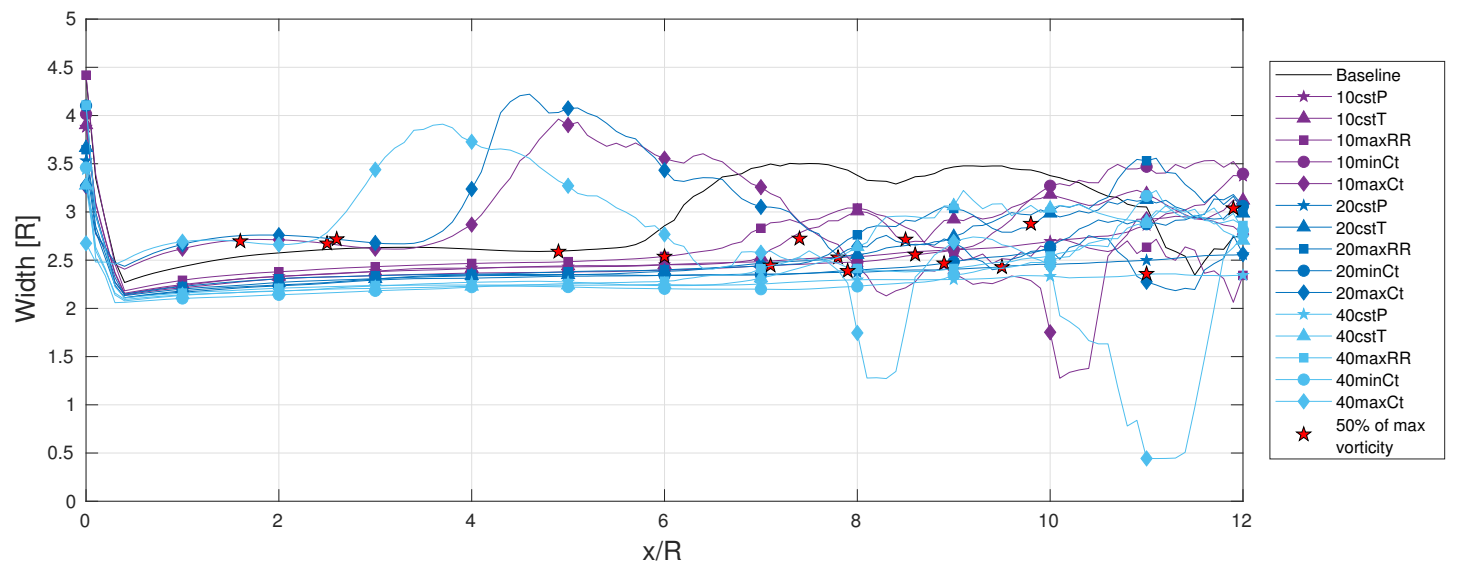

Figure 11. The smoothed wake width determined from contours shown in Fig. 8 as twice the average distance from the centroid to the contour. Most cases show a very similar pattern of wake growth, but the maxCt and Baseline cases grow and fluctuate much more.

each $x$, we find the maximum vorticity between $50 \%$ and $150 \%$ of the rotor radius and that is marked with a small red dot. The red star represents the first $x$ location downstream of the rotor where the local maximum is less than or equal to $50 \%$ of the global maximum for that case. The precise definition is arbitrary, but it helps to indicate the rate of decreasing vorticity strength. How far downstream 50\% of the maximum vorticity occurs tracks with the qualitative observations made from the wake visualizations in Fig. 8 and we see that there is no significant wake decay until the vorticity significantly decays. In particular, we see that vorticity in the maxCt cases decays the fastest and that the maxRR method appears to have the predicted effect.

A final qualitative metric is shown in Fig. 11, which shows a calculation of wake width. This width was calculated from the same routine as the wake visualizations in Fig. 8 with the width taken as twice the average distance from a contour to its centroid. These widths as a function of $x$ are then smoothed for clarity in plotting. The same $x$ locations marking $50 \%$ of the maximum vorticity are also plotted for each case to show how it corresponds to the growth of the wake. The Baseline and maxCt cases all grow much faster at first with subsequent reductions suggesting wake decay. It should be noted that the contour selecting routine described above is a bit arbitrary and imperfect, especially when the flow becomes more chaotic, so larger fluctuations should be considered with caution. The rest of the cases stack up very neatly in order of derate with lower derates (higher power) producing wider wakes due to the larger velocity deficits and resulting shear layers.

\subsection{Quantitative results}

275 Figure 12 shows the results of all simulated cases in terms of the achieved $C_{P}, C_{t}$, and $C_{q}$, the coefficients of power, thrust, and torque, respectively. $C_{P}$ was defined previously, and $C_{t}$ and $C_{q}$ are defined as

$C_{t}=\frac{T}{0.5 \rho U_{\infty}^{2} A_{T}}$, 
https://doi.org/10.5194/wes-2021-122

Preprint. Discussion started: 19 November 2021

(c) Author(s) 2021. CC BY 4.0 License.

(c) (i)

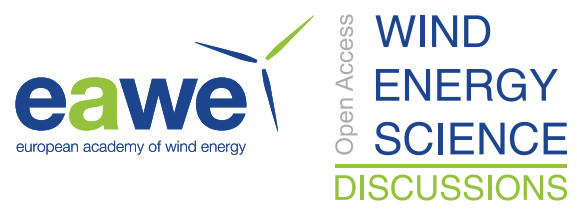

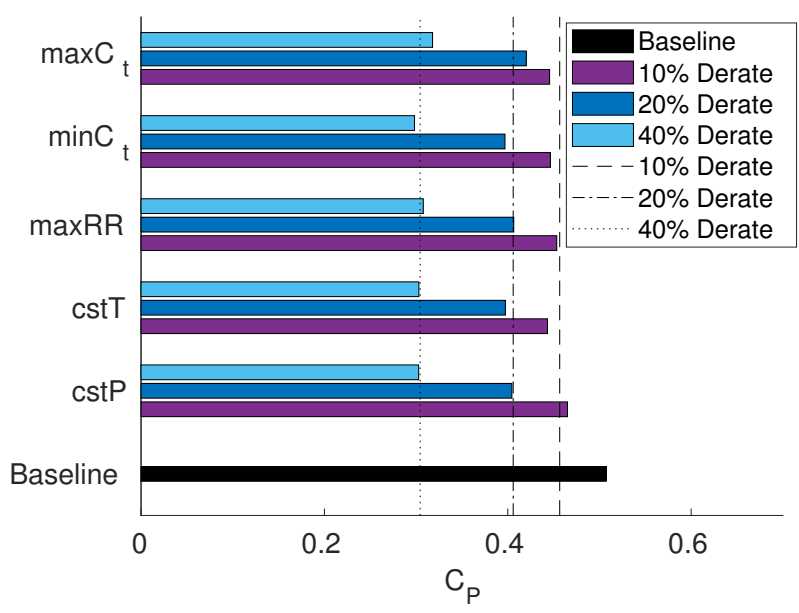

(a) The $C_{P}$ 's achieved in each simulation.

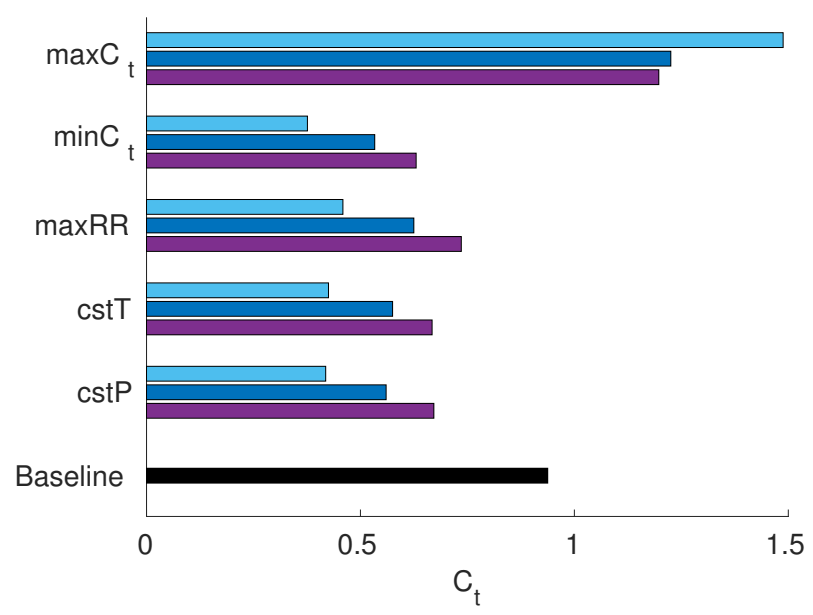

(b) The $C_{t}$ 's achieved in each simulation.

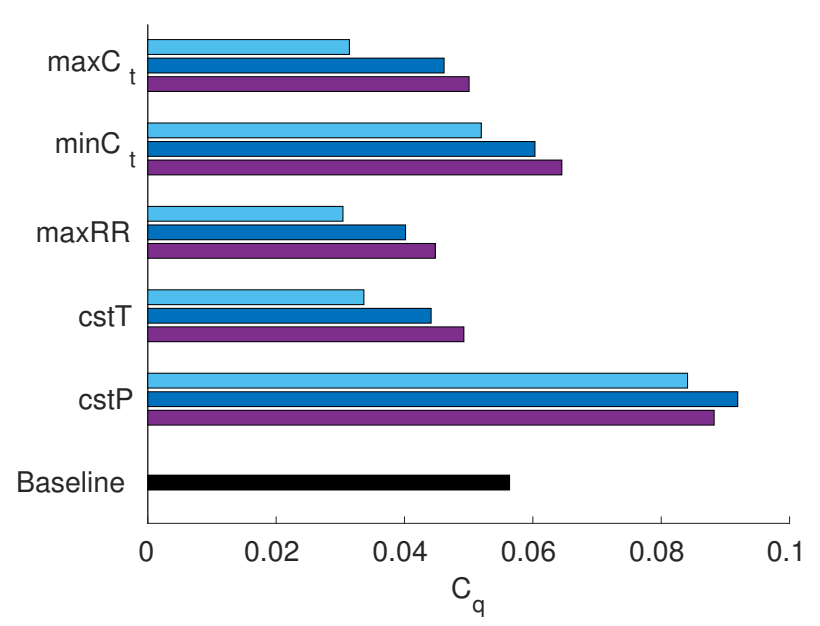

(c) The $C_{q}$ 's achieved in each simulation.

Figure 12. Plots of the resulting coefficients of power $\left(C_{P}\right)$, thrust $\left(C_{t}\right)$, and torque $\left(C_{q}\right)$ of each simulation show that the target derate was nearly achieved in each case, the much larger thrust in the maxCt cases, and the larger torques in the cstP cases. 


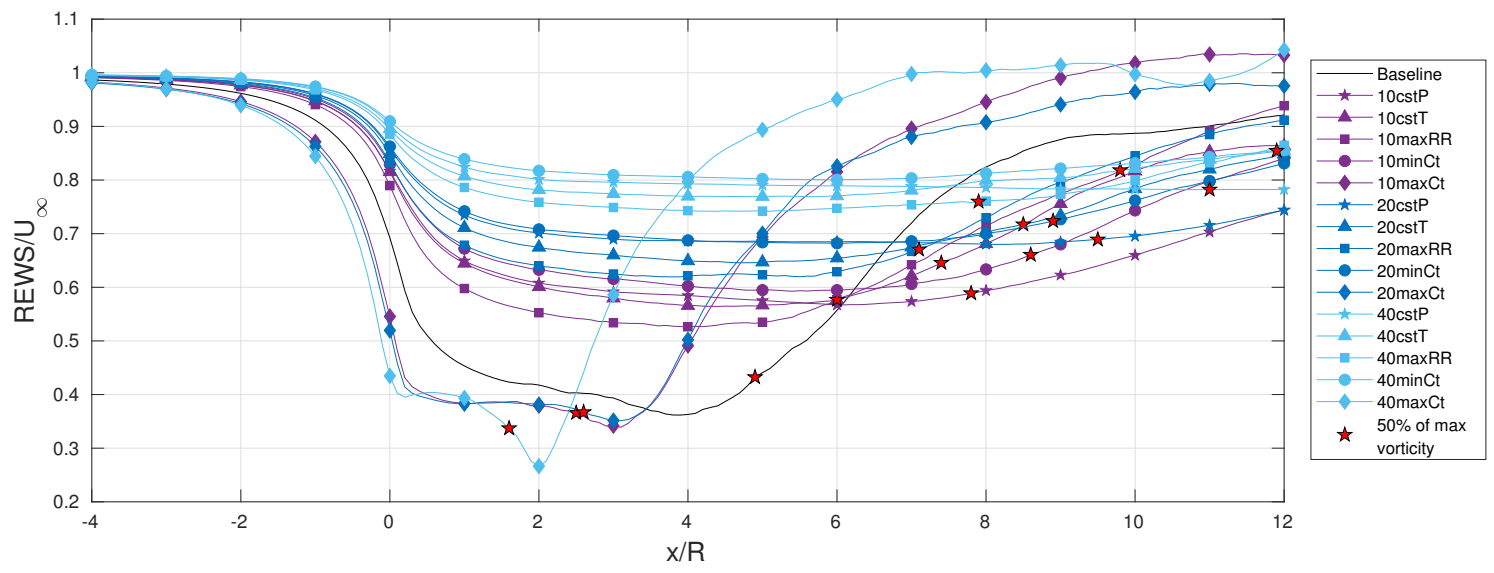

Figure 13. The rotor-equivalent wind speed provides an indication of velocity recovery, which largely correlates to the derate, though the maxCt and Baseline cases evolve very differently from the rest.

and

$C_{q}=\frac{Q}{0.5 \rho U_{\infty}^{2} A_{T} R}$,

where $T$ is the rotor thrust, $Q$ is the rotor torque, and $R$ is the rotor radius. In Fig. 12a, we see that many cases did not perfectly achieve the desired derate but that they are all very close. Figure $12 \mathrm{~b}$ shows first that all methods except maxCt result in reduced thrusts relative to the Baseline and that, in most cases, a greater derate corresponds to less thrust. Notably, the maxCt cases are the exception and their thrusts increase with greater derate. Finally, Fig. 12c shows the torque coefficient. Here, it is the constant pitch cases that stand out. As mentioned previously, because the Baseline operation is near the maximum TSR for the conditions, the TSR was reduced to derate the turbine while keeping the pitch constant. Since the turbine is operating at a lower speed, the torque is higher to achieve the desired power.

We can also evaluate the results through more quantitative metrics such as the rotor-equivalent wind speed, or $R E W S$, shown in Fig. 13. The REWS is calculated for the same rotor location as the simulated turbine at each $x$ as

$R E W S=\sqrt[3]{\frac{1}{A_{T}} \sum \bar{U}^{3} \Delta y \Delta z}$,

where $\bar{U}$ is the temporally averaged streamwise velocity, and the sum is taken over discrete $\Delta y$ and $\Delta z$ that fall on or within $A_{T}$ at each $x$. This provides a sense of the velocity recovery rate for each case. Here again, we see that the Baseline and maxCt cases stand out producing a larger drop in $R E W S$ in the near-wake because of their larger velocity deficits, but a much faster recovery thereafter. The maxCt cases are the only cases to fully recover to the freestream velocity. It must also be noted that the locations marking 50\% of the maximum vorticity correspond approximately to the beginning of the recovery phase of the wake after the initial decay in $R E W S$. In the near-wake, the other cases are organized, first, by derate with lower derates maintaining a higher $R E W S$ on account of having smaller deficits, and, second, by technique with minCt, cstP, cstT, and maxRR having 


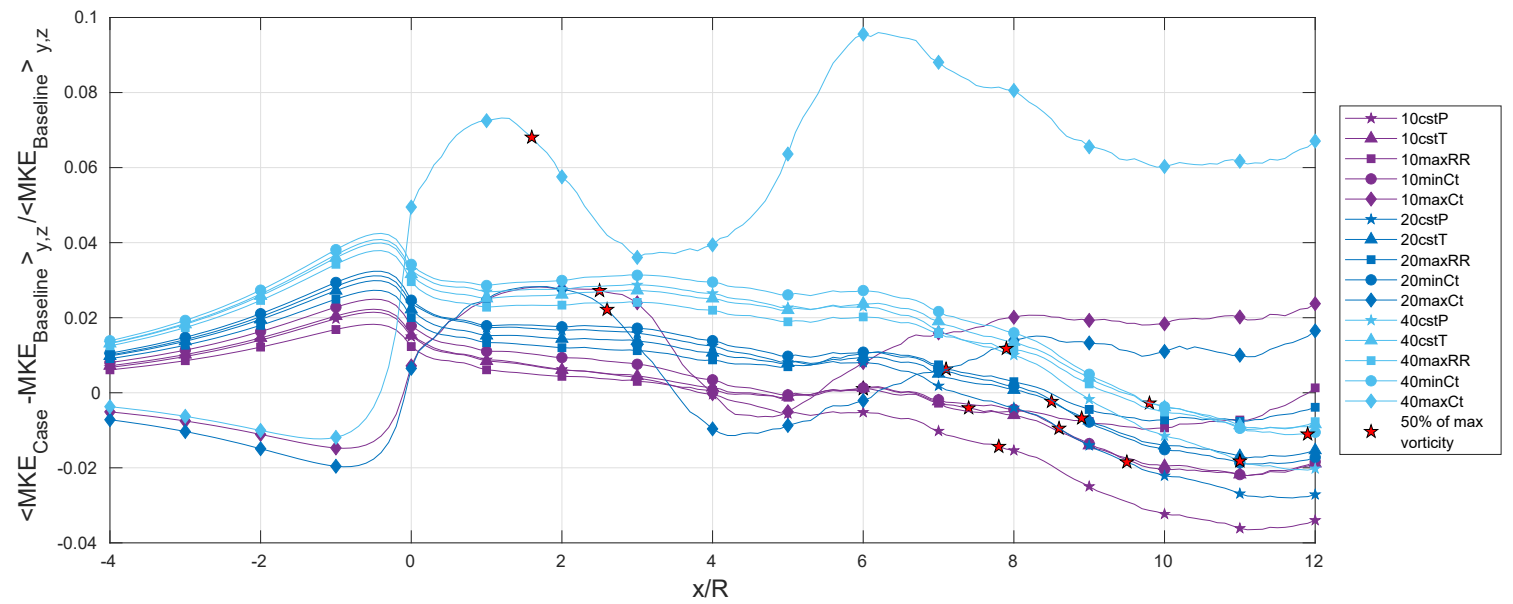

Figure 14. The excess energy, calculated as the spatially averaged difference between each case and the Baseline, shows how the evolution of energy in the domain differs among the cases.

decreasing $R E W S$ in that order within a derate. In the far-wake, some of the lower derates begin to exceed the $R E W S$ of higher derates, most notably the maxRR cases. This is the first suggestion of a break-even between producing a large velocity deficit and facilitating wake recovery by shear production.

Another way to view wake recovery is in terms of energy, and more specifically in terms of the excess energy relative to the Baseline case. Figure 14 shows the difference between the mean kinetic energy (MKE) of each case at each $x$ from the corresponding MKE of the Baseline spatially averaged in the $y-z$ plane and normalized by the spatially average MKE of the Baseline. It is essentially a percent difference in energy between each case and the Baseline as a function of $x$. The MKE is

$M K E=\frac{1}{2}\left(\bar{U}^{2}+\bar{V}^{2}+\bar{W}^{2}\right)$,

where $\bar{V}$ and $\bar{W}$ are the temporally averaged transverse and vertical velocities, respectively. It should be noted that this metric would be more meaningful if it were calculated across an area representing some physical parity such as a wake envelope or return to freestream velocity (similar to a boundary layer height) rather than spatially averaging across an entire plane. This gets at some of the inherent difficulty in defining a wind turbine wake, but this metric maintains consistency in that all cases use the same domain, so there is a parity in that. It is likely no surprise by now that the maxCt cases stand apart from the rest. Their large induction zones produce a deficit of excess energy upstream of the turbine, which is further evidence of their blockage effect, and, opposite to all other cases, there is an almost immediate increase in excess energy downstream of the turbine. Though the REWS calculation would suggest a reduced MKE, recall that it is only calculated within a rotor-swept area while this excess energy calculation includes the entire $y-z$ plane. Recalling Fig. 9, it is the acceleration of the freestream around the wake that causes the excess energy observed here. This effect, however, is short-lived, and the excess energy in the maxCt cases decays again before exhibiting another small increase that can be attributed to the increased mixing. All other 


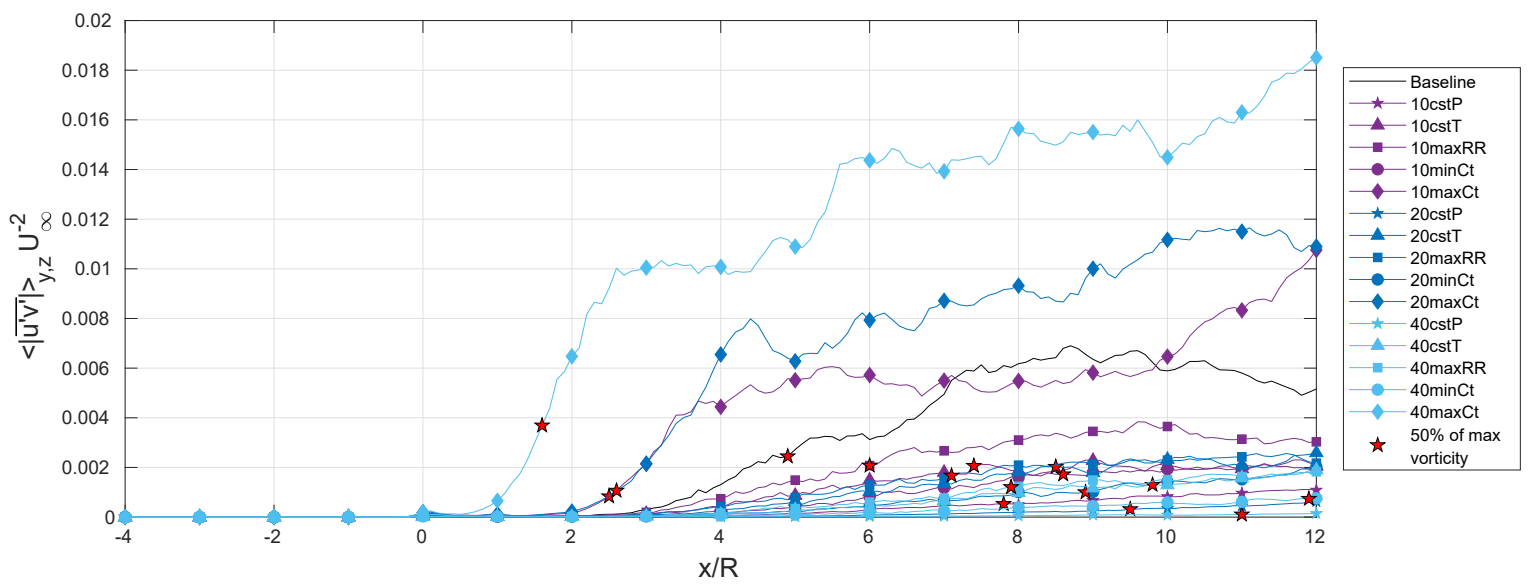

Figure 15. The absolute value of the Reynolds shear stress along the hub height centerline shows the stark difference in shear development between the maxCt and Baseline cases and all the other cases as well as the relation to vorticity breakdown.

cases are organized as they were in Fig. 13 and exhibit excess energy mostly by virtue of being derated relative to the Baseline. Interestingly, the other cases have a decreasing excess energy throughout the entirety of their wake recoveries and all have less energy relative to the Baseline by $5 \mathrm{D}$, which suggests reduced mixing relative to the Baseline.

The mixing between wake and ambient flow is well-represented by the Reynolds shear stress, which is plotted in Fig. 15 as a function of $x$. Again, in lieu of a well-defined wake envelope, the shear is spatially averaged over each $y-z$ plane. Most cases exhibit only mild shear within the domain length, but the maxCt cases produce substantially more shear than all cases including the Baseline. Notice again the effect of increased thrust in accelerating shear production as the higher derates in the maxCt cases produce more shear. In Fig. 16, contours of the difference in Reynolds shear stress between each case and the Baseline in the $y-z$ plane at 5D downstream reveal the spatial distribution of shear. Here we see that, not only do the maxCt cases produce greater shear, it has also penetrated the wake and does not remain along the shear layer typically along the edges of the wake envelope. This indicates that the maxCt cases have achieved net entrainment of energy into their wakes by $5 \mathrm{D}$ downstream while all other cases lag behind. Furthermore, we see that while $40 \mathrm{maxCt}$ had substantially larger spatially averaged shear, it does not appear to be as concentrated within the rotor disc area as it is for $10 \mathrm{maxCt}$ and $20 \mathrm{maxCt}$. We can also notice the 10maxRR appears to be close behind the maxCt cases in this respect, perhaps due in part to its accelerated vorticity breakdown. 

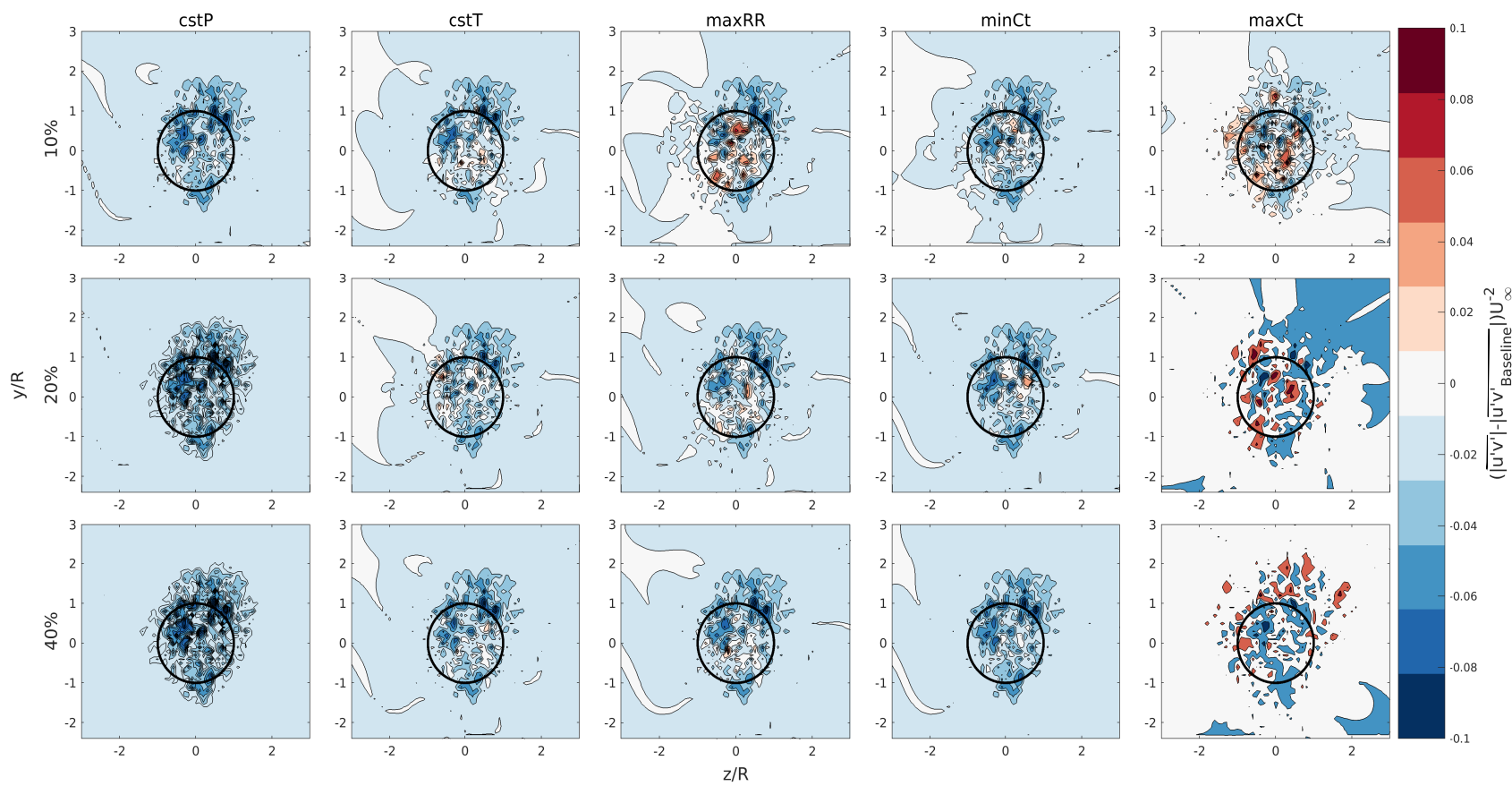

Figure 16. Contours of the difference of Reynolds shear stress between each case and the Baseline at five diameters downstream show the spatial distribution of shear and that it has penetrated into the rotor swept area (black circles) in the maxCt cases.

\subsection{Virtual rotor analysis}

To better say what the effects of the different derates and techniques would have on a downstream turbine, a virtual rotor is created. Figures 17 and 18 show the combined power of the simulated turbine and one in its wake normalized by the same using the Baseline. Since CACTUS is restricted to simulating a single turbine, the combined power of two turbines was estimated by calculating the power a turbine would produce at a given location based on the $R E W S$ calculated at that location. Specifically, for a rotor-swept area equal to the simulated turbine's, the $R E W S$ was calculated and then used to calculate the power of a virtual turbine at any location in the domain as

$P= \begin{cases}C_{P} \frac{1}{2} \rho U_{R E W S}^{3} A_{T}, & U_{R E W S}>4 \mathrm{~m} / \mathrm{s} \\ 0, & \text { otherwise, }\end{cases}$

where $4 \mathrm{~m} / \mathrm{s}$ is the rotor's cut-in speed and $C_{P}$ is taken as 0.46 , the rotor's theoretical coefficient of power at the range of wind speeds found in the wake. Considering Fig. 17, we see that, due to their relatively faster recovery, the maxCt cases produce more total power than other cases. In particular, 10maxCt produces more total power than the Baseline from about 2.5D and onward reaching a peak of almost 1.2 at 3D. From 4-6D, 10maxCt is approximately constant at about 1.1 times the 


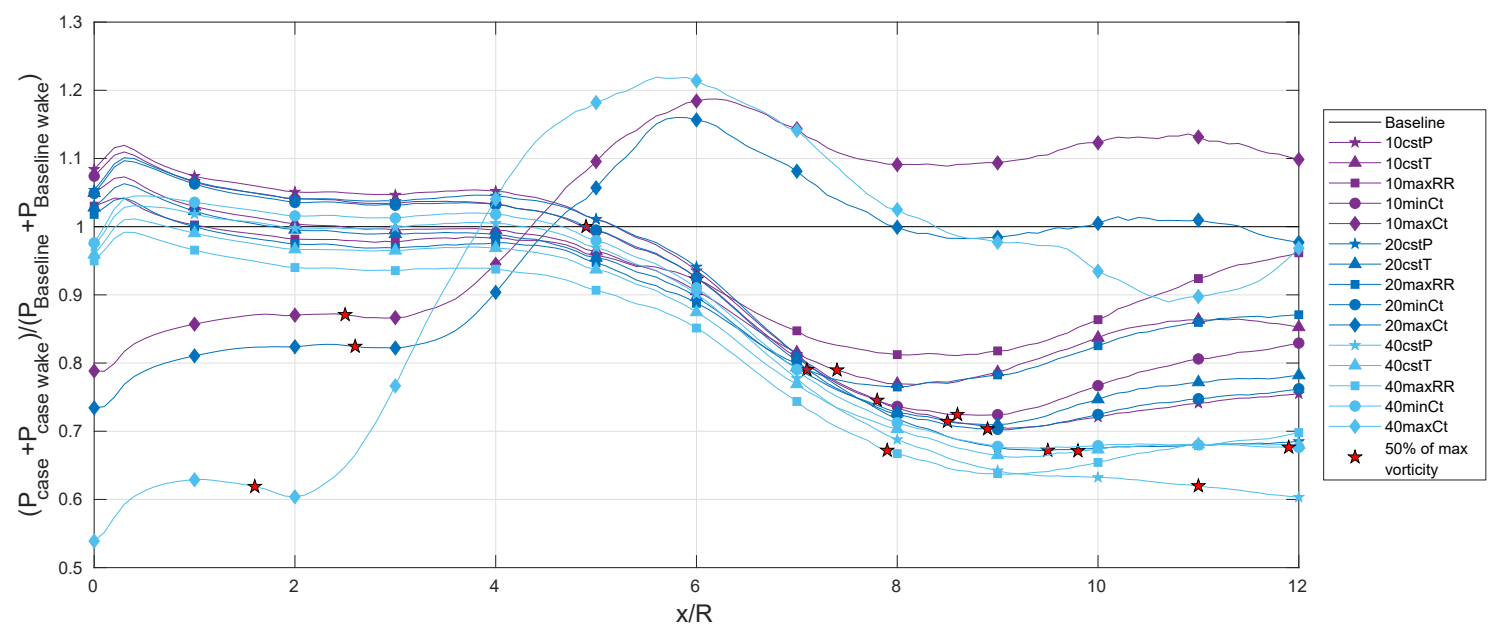

Figure 17. The total power of each case and the power of another turbine operating optimally aligned and in its wake as a function of $x$ shows that higher derates offer little to no advantage and that the maxCt cases perform best of all techniques.

power produced in the Baseline. Curiously, the $20 \%$ and $40 \%$ derates with maxCt end up at about the same total power at 6D downstream despite taking very diffent paths, especially in the near-wake. Indeed, 40maxCt technically produces the highest total power of all cases, but it is not sustained. This suggests a balance between maximizing thrust and sacrificing power to a greater derate. That is, the added thrust achievable with a $40 \%$ derate produced wake dyanmics that made up for the initial power lost compared to a $20 \%$ derate with a lower thrust. While it does not surpass the Baseline, 10maxRR is best among the remaining cases and could, perhaps, exceed the Baseline given more distance downstream.

Finally, Fig. 18 shows the same total power calculation, but now as a function of $z$ at 5D downstream. In this view, 10maxCt is still the best choice at practically all $z$ 's and best when aligned with the upstream turbine. This result may appear unexpected given previous studies that showed that aligned turbines may not be well positioned to benefit from AIC (Annoni et al., 2015), but it is directly in line with those predictions. Because the maxCt cases produce wakes that are recovered enough for the excess energy from derating to have penetrated the wake, the best position for a downstream turbine in these cases is aligned with the upstream turbine. This is also fortuitous since it is the position for which wake mitigation is most needed. In contrast, all other cases suggest a peak total power for a downstream turbine that is offset transversely from the upstream turbine, which is exactly what was shown by Annoni et al. (2015). 
https://doi.org/10.5194/wes-2021-122

Preprint. Discussion started: 19 November 2021

(c) Author(s) 2021. CC BY 4.0 License.

(c) (i)
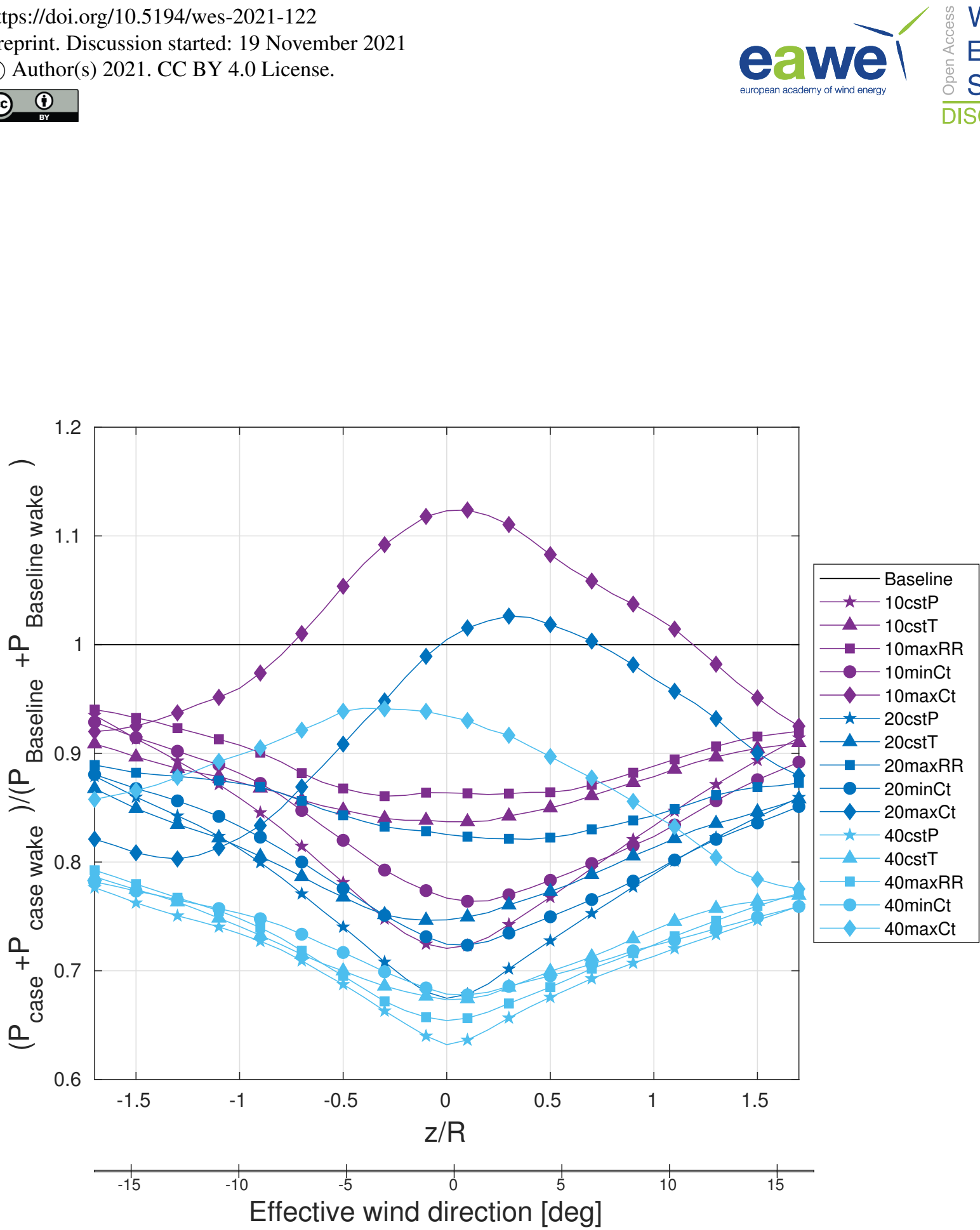

Figure 18. The total power of each case and another turbine operating optimally in its wake at different spanwise locations and effective wind directions at five diameters downstream shows that the highest combined power is achieved with a downstream turbine aligned with an upstream turbine operating with maximized thrust at a $10 \%$ derate. 


\subsection{Loads}

A final and important consideration is the effect non-standard turbine operation may have on loads. The out-of-plane (OOP) (i.e., not necessarily the result of only forces normal to the blade but perpendicular to the rotor plane) and in-plane (IP) bending moments for the simulated turbine and the OOP bending moments for the virtual turbines were calculated and are shown in Figures 19. For the simulated turbine, CACTUS outputs allow the moments to be calculated as

$M_{O O P, s i m}=C_{t}(r) \frac{1}{2} \rho \pi R^{2} U_{\infty}^{2} \frac{2 R}{3 B}$,

$M_{I P, s i m}=C_{q}(r) \frac{1}{2} \rho \pi R^{2} U_{\infty}^{2} / B$

where $B$ is the number of blades and $C_{t}$ and $C_{q}$ are evaluated as functions of rotor radius (Manwell et al., 2009).

For the virtual turbine, the OOP moment was estimated by using a control volume approach to derive the forces over the rotor-swept area. At 5D, the temporally averaged streamwise velocity was extracted from the 10 grid points corresponding to a blade pointed straight up, down, left, or right and these 40 points were averaged to provide an approximately azimuthally averaged streamwise velocity for a rotor area aligned with the simulated turbine and freestream flow. Because the pressure difference created by an actual rotor would be significantly larger than the pressure difference across the virtual rotor, the pressure difference was not included. The OOP moment was then calculated as

$M_{O O P, v i r}=\sum_{r=0}^{r=R} \frac{1}{2} \rho \overline{U(r)}^{2} A_{E}(r) r$,

where $A_{E}(r)$ are the span-dependent element areas approximated as the product of the element span length and the chord length at the center of each element and $r$ is the spanwise distance along the rotor.

Looking first at the simulated turbine (Figures 19a and 19b), the price for increasing thrust is an approximately $25 \%$ increase in the OOP moment. All other cases would decrease the OOP moment since they also decrease thrust and moreso for higher derates. The IP moments correlate to the results for $C_{q}$ shown in Fig. 12c as expected. For the cstP cases to produce the given power without adjusting pitch, the $T S R$ must be very low, which requires a compensation in torque. It can also be inferred from Fig. 12b and Fig. 19a that the tower fore-aft moment would increase by around 25\% as it is proportional to the turbine thrust. The OOP moments of the virtual turbine (Fig. 19c) indicate that it would experience an approximately $50 \%$ increase in moments during the maxCt cases. Since these blade moments are produced by forces in the streamwise direction, the virtual turbine would likely also experience a similar increase in tower fore-aft moment. There is obvious uncertainty in the method for computing the OOP moments of the virtual turbine, which is why they have been normalized by the Baseline rather than showing the magnitudes that were calculated. It is interesting as well that there is no clear trend between derate and moments for the virtual turbine. Finally, the likely fatigue loading can be compared among the cases by using the turbulence intensity (TI) as a proxy as it is the cause of most fatigue (Kanev et al., 2018). This is done by averaging the streamwise turbulence intensity across a rotor-swept area at 5D downstream as shown in Fig. 19d. The higher $T I$ in the maxCt cases again suggests 
https://doi.org/10.5194/wes-2021-122

Preprint. Discussion started: 19 November 2021

(c) Author(s) 2021. CC BY 4.0 License.

(c) (i)

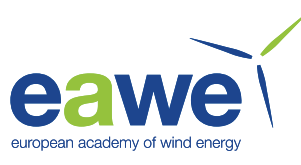

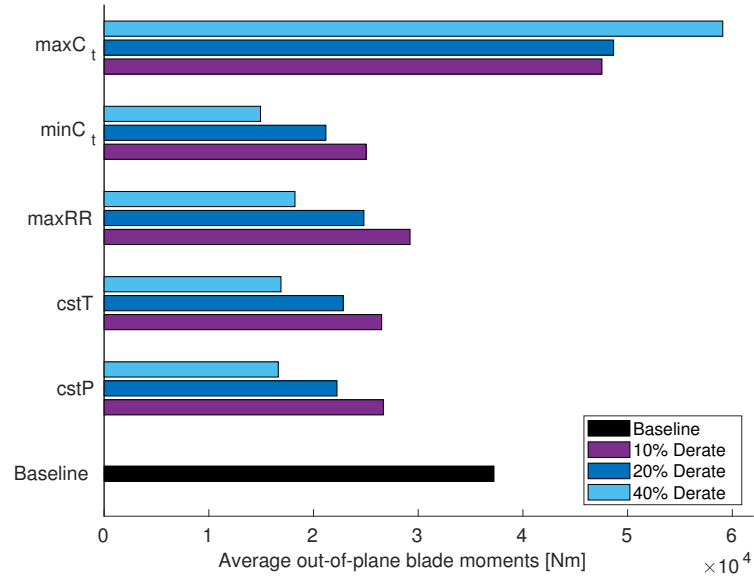

(a) Out-of-plane bending moments of the upstream, simulated turbines.

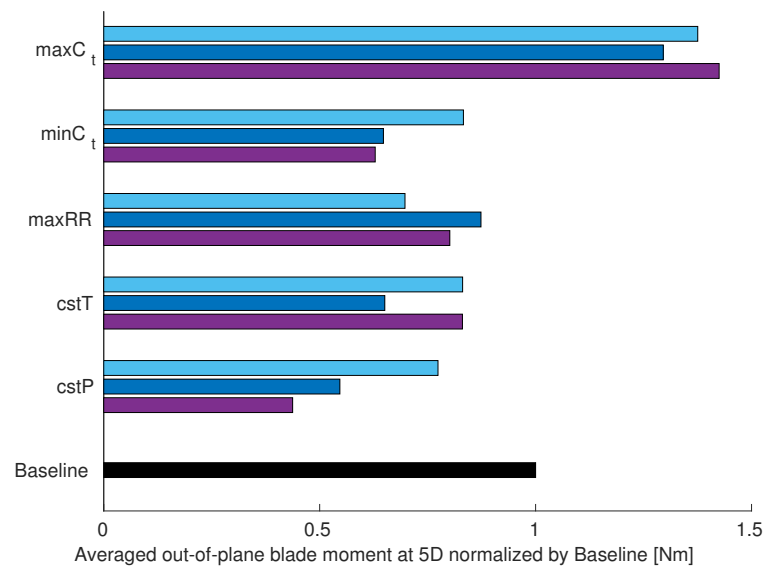

(c) Out-of-plane bending moments of a virtual turbine at 5D.

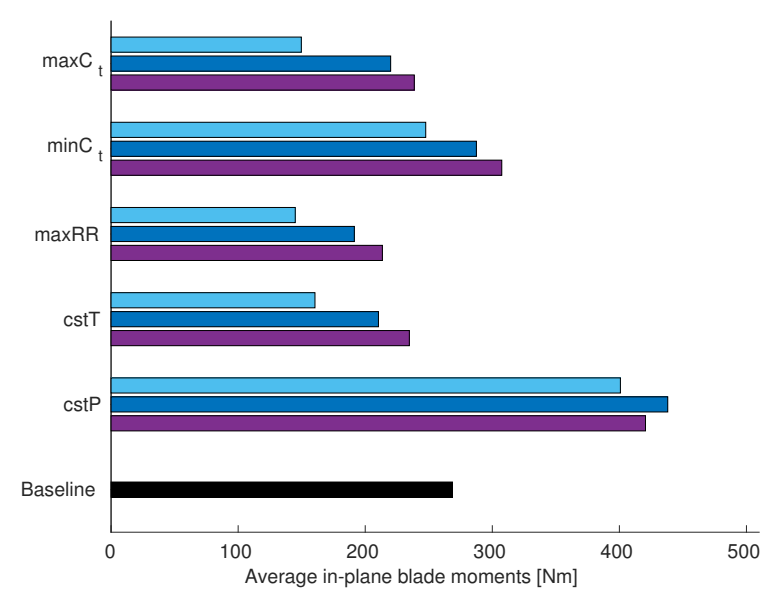

(b) In-plane bending moments of the upstream, simulated turbines.

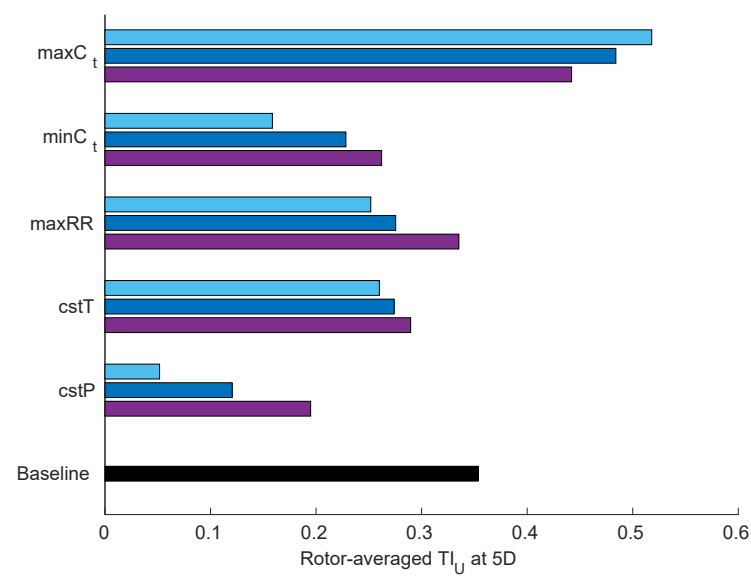

(d) Rotor-averaged streamwise turbulence intensity of a virtual turbine at $5 \mathrm{D}$.

Figure 19. Out-of-plane and in-plane bending moments of the upstream, simulated turbines and out-of-plane moments of virtual turbines at 5D downstream show that maximizing the thrust will increase loads on both turbines. The rotor-averaged turbulence intensity suggests higher fatigue loading as a result too. 
https://doi.org/10.5194/wes-2021-122

Preprint. Discussion started: 19 November 2021

(c) Author(s) 2021. CC BY 4.0 License.

\section{(c) (i)}

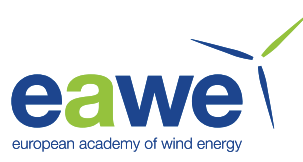

that there is a price to pay in added fatigue loading. The cost of an increase in combined power output of the two turbines considered may be an increase in loads in both.

\section{Discussion}

Within the limitations of the simulations, these results suggest that operating an upstream turbine at a small derate and maximized thrust could increase the total power of it and a downstream turbine by over $10 \%$ of a Baseline, conventional, operation with streamwise turbine spacing of about $4 \mathrm{D}$ to at least $6 \mathrm{D}$. While many previous studies have identified minimum thrust as the optimal method (Mirzaei et al., 2015; Pedersen and Larsen, 2020; Schaak, 2006; Vitulli et al., 2019), several have also found that overinduction (increasing thrust) produces higher total power than underinduction (Munters and Meyers, 2016, 2018; Cossu, 2021; Dilip and Porté-Agel, 2017). The latter studies, however, use very different methods both in the details of the derating and the simulations so it is difficult to compare their results to these.

It remains to be seen if the positive effects of high thrust can be carried on farther downstream and/or across additional rows of turbines, or if this would require something like a cascade of derates with higher derates upstream decreasing to conventional operation at the last turbine downstream as has been previously suggested (Bitar and Seiler, 2013; Corten and Schaak, 2004). Though the domain does not extend as far as typical transverse turbine spacing, Fig. 18 suggests that this method works best when the turbines are aligned, which is contrary to conventional siting in which turbines are offset with respect to the dominant wind direction. This is, however, in agreement with when wake mitigation techniques such as AIC are most relevant, i.e., when turbines are aligned with the wind and subject each other to their wakes. It should also be noted that only the maxCt cases have peak combined power outputs around $z / R=0$ while all other cases appear to have peaks at least 1.3D away from $z / R=0$, which is in agreement with results from Annoni et al. (2015). Indeed, Fig. 16 reinforces the importance of sufficient wake mixing when turbines are aligned such that the excess energy generated by derating can penetrate the wake and be captured by the downstream turbine.

The mechanism by which this improvement works appears to be the effective blockage that is created by higher turbine thrust forces. The high thrust rapidly slows the flow passing through the rotor, and, due to conservation, the volume it occupies expands. Likewise, this forces the ambient flow to accelerate around the near-wake setting up a high gradient and rapid increase in Reynolds shear stress. This in turn aids in mixing between the ambient flow and wake facilitating faster recovery. It also appears that this process is intimately connected to the decay of the tip vortices. Their decay is likely aided by the turbulence that is generated by the high gradient across the near-wake, which in turn allows for greater mixing into the wake. Finally, there

415 is an apparent connection between the degree of derate and the increase in thrust. The 10maxCt case was the only one of the maxCt cases whose wake recovery could compensate for the derate. The precise balance between these variables and whether or not thrust needs to be maximized or just increased should be explored in future studies.

The verification study suggests that cases with higher thrust may benefit from simulating more revolutions for better convergence, though the average residuals of the energy balance remained small. The virtual rotor method is also imperfect, in part because it does not capture the feedback between the turbine and its inflow through its induction zone. The biggest limitation 
of CACTUS, however, is the lack of turbulence coming from the inflow and it is unclear how ambient turbulence would affect the results. On the one hand, it may diminish the affect of the induced turbulence created by high thrust. On the other hand, it has been shown that turbine power production increases with turbulence intensity, so it is possible that the addition of turbulence would allow the maxCt cases to produce even more combined power relative to the other methods (Hansen et al., 2012; Talavera and Shu, 2017). The benefits of increased total power using this method need to be weighed against the increase in loads it is likely to cause.

\section{Conclusions}

Sixteen simulations were performed using a mid-fidelity FVWM code to analyze the effects of five different methods of AIC at three different derates against a Baseline. Though the results are limited by the method, they strongly suggest that operating an upstream turbine at a derate of $10 \%$ while maximizing its thrust can increase the combined power of it and an aligned turbine by about $10 \%$ at a streamwise turbine spacing of about $4 \mathrm{D}$ to at least $6 \mathrm{D}$. In other words, the total power compensates for the $10 \%$ derate and produces an additional 10\% compared to operating both turbines conventionally. The increased thrust quickly establishes a steep gradient in flow velocities across the boundaries of the turbine wake, which destablizes the tip vortices and rapidly mixes to the interior of the wake. Both the upstream and downstream turbines may experience higher loads as a result. Future studies should consider confirming these results with higher fidelity methods including the effects of different turbulence levels, multiple turbines, and a more detailed study of the effect on loads.

Data availability. Forthcoming upon final submission

Author contributions. DH is responsible for conceptualization, methodology, formal analysis, visualization, and writing of the original draft. $\mathrm{DM}$ is responsible for methodology and reviewing the original draft. CK is responsible for funding acquisition, supervision, and reviewing the original draft.

Competing interests. The authors declare that they have no conflict of interests.

Acknowledgements. This paper describes objective technical results and analysis. Any subjective views or opinions that might be expressed in the paper do not necessarily represent the views of the U.S. Department of Energy or the United States Government. Sandia National Laboratories is a multi-mission laboratory managed and operated by National Technology \& Engineering Solutions of Sandia, LLC, a 
https://doi.org/10.5194/wes-2021-122

Preprint. Discussion started: 19 November 2021

(C) Author(s) 2021. CC BY 4.0 License.

(c) (i)

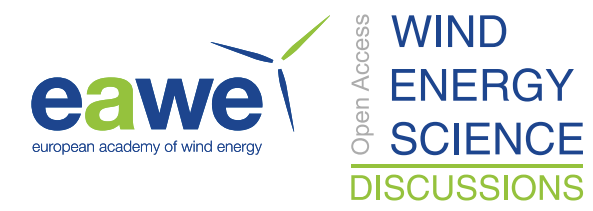

wholly owned subsidiary of Honeywell International Inc., for the U.S. Department of Energy's National Nuclear Security Administration under contract DE-NA0003525. 
https://doi.org/10.5194/wes-2021-122

Preprint. Discussion started: 19 November 2021

(c) Author(s) 2021. CC BY 4.0 License.

(c) (i)

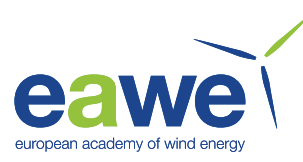

\section{References}

Ananthan, S., Lieshman, J. G., and Ramasamy, M.: The role of filament stretching in the free-vortex modeling of rotor wakes, 58th Annual

Forum and Technology Display of the American Helicopter Society International, https://doi.org/10.1093/ajae/aau104, 2002.

Annoni, J., Gebraad, P. M. O., Scholbrock, A. K., Fleming, P., and van Wingerden, J. W.: Analysis of axial-induction-based wind plant control using an engineering and a high-order wind plant model, Wind Energy, 19, 1135-1150, https://doi.org/10.1002/we, 2015.

Berg, J., Bryant, J., Leblanc, B., Maniaci, D., Naughton, B., Paquette, J., Resor, B., White, J., and Kroeker, D.: Scaled Wind Farm Technology Facility Overview, Tech. rep., 2013.

Bhagwat, M. J. and Leishman, J. G.: Time-accurate modeling of rotor wakes using a free-vortex wake method, 18th Applied Aerodynamics Conference, pp. 236-246, https://doi.org/10.2514/6.2000-4120, 2000.

Bhagwat, M. J. and Leishman, J. G.: Stability, consistency and convergence of time-marching free-vortex rotor wake algorithms, Journal of the American Helicopter Society, 46, 59-71, https://doi.org/10.4050/jahs.46.59, 2001.

Bitar, E. and Seiler, P.: Coordinated Control of a Wind Turbine Array for Power Maximization, in: 2013 American Control Conference (ACC), pp. 2898-2904, https://doi.org/10.1109/ACC.2013.6580274, 2013.

Branlard, E., Papadakis, G., Gaunaa, M., Winckelmans, G., and Larsen, T. J.: Aeroelastic large eddy simulations using vortex methods: Unfrozen turbulent and sheared inflow, Journal of Physics: Conference Series, 625, https://doi.org/10.1088/1742-6596/625/1/012019, 2015.

Brown, K., Houck, D., Maniaci, D., Westergaard, C., and Laboratories, S. N.: Rapidly Recovering Wind Turbine Wakes with Dynamic Pitch and Rotor Speed Control, in: AIAA SciTech Forum, January, pp. 1-16, https://doi.org/10.2514/6.2021-1182, 2021.

Castillo, L. and Newman, A. J.: Examination of the spatially developing wind turbine boundary layer: Low dimensional analysis, large scales of motion and energy entrainment, 43rd Fluid Dynamics Conference, pp. 1-11, https://doi.org/10.2514/6.2013-3120, 2013.

Chamorro, L. P. and Porté-Agel, F.: A Wind-Tunnel Investigation of Wind-Turbine Wakes: Boundary-Layer Turbulence Effects, BoundaryLayer Meteorology, 132, 129-149, https://doi.org/10.1007/s10546-009-9380-8, 2009.

Chamorro, L. P., Guala, M., Arndt, R., and Sotiropoulos, F.: On the evolution of turbulent scales in the wake of a wind turbine model, Journal of Turbulence, 13, N27, https://doi.org/10.1080/14685248.2012.697169, 2012.

Chamorro, L. P., Arndt, R. E. a., and Sotiropoulos, F.: Turbulence within variable-size wind turbine arrays, Journal of Physics: Conference Series, 555, 012 098, https://doi.org/10.1088/1742-6596/555/1/012098, 2014.

Churchfield, M. J. and Lee, S.: SOWFA, 2012.

475 Corniglion, R., Harris, J., Peyrard, C., and Capaldo, M.: Comparison of the free vortex wake and actuator line methods to study the loads of a wind turbine in imposed surge motion, Journal of Physics: Conference Series, 1618, 052 045, https://doi.org/10.1088/17426596/1618/5/052045, 2020.

Corten, G. P. and Schaak, P.: More Power and Less Loads in Wind Farms: 'Heat and Flux', in: European Wind Energy Conference, 2004.

Cossu, C.: Wake redirection at higher axial induction, Wind Energy Science Discussions, 6, 1-12, https://doi.org/10.5194/wes-2020-111, 2021.

Dilip, D. and Porté-Agel, F.: Wind Turbine Wake Mitigation through Blade Pitch Offset, Energies, 10, https://doi.org/10.3390/en10060757, 2017.

Ennis, B. L., Kelley, C. L., and Maniaci, D. C.: Dynamic wake meandering model comparison with varying fidelity models for wind turbine wake prediction, Annual Forum Proceedings - AHS International, 4, 2885-2894, 2015. 
https://doi.org/10.5194/wes-2021-122

Preprint. Discussion started: 19 November 2021

(C) Author(s) 2021. CC BY 4.0 License.

Hansen, K. S., Barthelmie, R. J., Jensen, L. E., and Sommer, A.: The impact of turbulence intensity and atmospheric stability on power deficits due to wind turbine wakes at Horns Rev wind farm, Wind Energy, 15, 183-196, https://doi.org/10.1002/we.512, 2012.

Houck, D. and Cowen, E. A. T.: Can you accelerate wind turbine wake decay with unsteady operation?, in: AIAA Scitech 2019 Forum, January, https://doi.org/10.2514/6.2019-2084, 2019.

Houck, D. R.: Review of wake management techniques for wind turbines, Wind Energy, pp. 1-26, https://doi.org/10.1002/we.2668, 2021.

Iungo, G. V., Viola, F., Camarri, S., Porté-Agel, F., and Gallaire, F.: Linear stability analysis of wind turbine wakes performed on wind tunnel measurements, Journal of Fluid Mechanics, 737, 499-526, https://doi.org/10.1017/jfm.2013.569, 2013.

Ivanell, S., Mikkelsen, R., Sorensen, J. N., and Henningson, D.: Stability analysis of the tip vortices of a wind turbine, Wind Energy, 13, 705-715, https://doi.org/10.1002/we, 2010.

Kanev, S., Savenije, F. J., and Engels, W.: Active wake control: An approach to optimize the lifetime operation of wind farms, Wind Energy, 21, 488-501, https://doi.org/10.1002/we.2173, 2018.

Katic, I., Hojstrup, J., Jensen, N. O.: A simple model for cluster efficiency, European wind energy conference and exhibition, pp. 407-410, 1986.

Kelley, C. L.: Aerodynamic Design of the National Rotor Testbed, Tech. Rep. SAND2015-8989, Sandia National Laboratories, 2015.

Kelley, C. L. and Ennis, B. L.: SWiFT site atmospheric characterization, Tech. Rep. January, https://doi.org/10.2172/1237403, 2016.

Kelley, C. L., Maniaci, D. C., and Resor, B. R.: Horizontal-axis wind turbine wake sensitivity to different blade load distributions, in: 33rd Wind Energy Symposium, January, https://doi.org/10.2514/6.2015-0490, 2015.

Kelley, C. L., Maniaci, D. C., and Resor, B. R.: Scaled Aerodynamic Wind Turbine Design for Wake Similarity, 34th Wind Energy Symposium, pp. 1-28, https://doi.org/10.2514/6.2016-1521, 2016.

Kheirabadi, A. C. and Nagamune, R.: A quantitative review of wind farm control with the objective of wind farm power maximization, Journal of Wind Engineering \& Industrial Aerodynamics, 192, 45-73, https://doi.org/10.1016/j.jweia.2019.06.015, 2019.

Leishman, J. and Beddoes, T.: A Semi-Empircial Model for Dynamic Stall, Journal of the American Helicopter Society, 34, https://doi.org/10.4050/jahs.35.3.3, 1989.

Leishman, J. G., Bhagwat, M. J., and Bagai, A.: Free-vortex filament methods for the analysis of helicopter rotor wakes, Journal of Aircraft, 39, 759-775, https://doi.org/10.2514/2.3022, 2002.

510 Lignarolo, L. E. M., Ragni, D., Scarano, F., Simão Ferreira, C. J., van Bussel, G. J. W., and Ferreira, C. J. S.: Tip-vortex instability and turbulent mixing in wind-turbine wakes, Journal of Fluid Mechanics, 781, 467-493, https://doi.org/10.1017/jfm.2015.470, 2015.

Manwell, J., McGowan, J., and Rogers, A. L.: Wind energy explained - theory, design and application, vol. 2, John Wiley \& Sons Ltd., 2nd edn., 2009.

Marten, D., Wendler, J., Pechlivanoglou, G., Nayeri, C. N., and Paschereit, C. O.: QBlade: An open source tool for design and simulation of horizontal and vertical axis wind turbines, International Journal of Emerging Technology and Advanced Engineering, 3, 264-269, https://doi.org/10.1017/S0140525X00034634, 2013.

Marten, D., Lennie, M., Pechlivanoglou, G., Nayeri, C. N., and Paschereit, C. O.: Implementation, Optimization, and Validation of a Nonlinear Lifting Line-Free Vortex Wake Module Within the Wind Turbine Simulation Code QBLADE, Journal of Engineering for Gas Turbines and Power, 138, 1-10, https://doi.org/10.1115/1.4031872, 2016.

Marten, D., Paschereit, C. O., Huang, X., Meinke, M., Schröder, W., Müller, J., and Oberleithner, K.: Predicting Wind Turbine Wake Breakdown Using a Free Vortex Wake Code, AIAA Journal, 58, 1-14, https://doi.org/10.2514/1.j058308, 2020. 
https://doi.org/10.5194/wes-2021-122

Preprint. Discussion started: 19 November 2021

(c) Author(s) 2021. CC BY 4.0 License.

(c) (1)

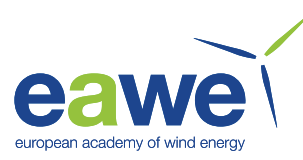

Mirzaei, M., Tuhfe, G., Giebel, G., Sorensen, P. E., and Poulsen, N. K.: Turbine control strategies for wind farm power optimization, in: American Control Conference, July, https://doi.org/10.1109/ACC.2015.7170979, 2015.

Montgomerie, B.: Methods for root effects,tip effects and extending the angle of attack range to $+/-100^{\circ}$, with application to aerodynamics for blades on wind turbines and propellers, Tech. Rep. FOI-R-1035-SE, Swedish Defense Research Agency, 2004.

Munters, W. and Meyers, J.: Effect of wind turbine response time on optimal dynamic induction control of wind farms, in: Journal of Physics: Conference Series, 753, https://doi.org/10.1088/1742-6596/753/5/052007, 2016.

Munters, W. and Meyers, J.: Optimal dynamic induction and yaw control of wind farms : effects of turbine spacing and layout, in: Journal of Physics: Conference Series, 2018.

530 Murray, J. and Barone, M.: The Development of CACTUS, a Wind and Marine Turbine Performance Simulation Code, https://doi.org/10.2514/6.2011-147, 2011.

Murray, J. C., Barone, M., and Chiu, P. K.: CACTUS User Guide.

NREL: FLORIS, https://github.com/NREL/floris, 2020.

Pedersen, M. and Larsen, G.: Integrated wind farm layout and control optimization, Wind Energy Science Discussions, in review, 1-19, https://doi.org/10.5194/wes-2020-31, 2020.

Quon, E. W., Doubrawa, P., and Debnath, M.: Comparison of Rotor Wake Identification and Characterization Methods for the Analysis of Wake Dynamics and Evolution, Journal of Physics: Conference Series, 1452, https://doi.org/10.1088/1742-6596/1452/1/012070, 2020.

Schaak, P.: Heat \& Flux: Enabling the Wind Turbine controller, Tech. Rep. ECN-E-06-017, Energy Research Centre of the Netherlands, 2006.

540 Talavera, M. and Shu, F.: Experimental study of turbulence intensity influence on wind turbine performance and wake recovery in a low-speed wind tunnel, Renewable Energy, 109, 363-371, https://doi.org/10.1016/j.renene.2017.03.034, 2017.

Vitulli, J. A., Larsen, G. C., Pedersen, M. M., Ott, S., and Friis-Møller, M.: Optimal open loop wind farm control, in: Journal of Physics: Conference Series, vol. 1256, https://doi.org/10.1088/1742-6596/1256/1/012027, 2019.

Wosnik, M., Bachant, P., Neary, V. S., and Murphy, A. W.: Evaluation of Design \& Analysis Code , CACTUS , for Predicting Cross-flow Hydrokinetic Turbine Performance, 2016.

Xu, B., Liu, B., Cai, X., Yuan, Y., Zhao, Z., and Wang, Y.: Accuracy of the aerodynamic performance of wind turbines using vortex core models in the free vortex wake method, Journal of Renewable and Sustainable Energy, 11, https://doi.org/10.1063/1.5121419, 2019.

Yang, X., Sotiropoulos, F., Conzemius, R. J., Wachtler, J. N., and Strong, M. B.: Large-eddy simulation of turbulent flow past wind turbines/farms: the Virtual Wind Simulator (VWiS), Wind Energy, 18, 2025-2045, https://doi.org/10.1002/we, 2015. 